\title{
THE GEISSER-LEVINE METHOD REVISITED AND ALGEBRAIC CYCLES OVER A FINITE FIELD
}

\author{
BRUNO KAHN
}

\begin{abstract}
We reformulate part of the arguments of T. Geisser and M. Levine relating motivic cohomology with finite coefficients to truncated étale cohomology with finite coefficients [9, 10]. This reformulation amounts to a uniqueness theorem for motivic cohomology, and shows that the Geisser-Levine method can be applied generally to compare motivic cohomology with other types of cohomology theories. We apply this to prove an equivalence between conjectures of Tate and Beilinson on cycles in characteristic $p$ and a vanishing conjecture for continuous étale cohomology.
\end{abstract}

\section{Contents}

1. Functoriality of motivic cohomology 3

2. The Geisser-Levine method revisited 17

3. Application to algebraic cycles over $\mathbb{F}_{p} \quad 34$

References $\quad 38$

In this paper, we provide a rather simpler proof of the results of Suslin-Voevodsky [26] and Geisser-Levine [9, 10] relating motivic cohomology with finite coefficients to truncated étale cohomology with finite coefficients. Our interest in doing so is twofold. First, to try and clarify the strategy of the proofs. Second, to make it clear to what extent this strategy can be applied in other situations, allowing one to compare motivic cohomology with other cohomology theories. Indeed, Theorem 2.34 below gives necessary and sufficient conditions for a "cohomology theory" in a suitable sense to be isomorphic to a version of motivic cohomology.

Our main application is to a comparison between motivic cohomology and $l$-adic cohomology over finite fields ( $c f$. Theorem 3.4). Since this comparison is in characteristic $p$, we have to choose the methods of $[9,10]$ which don't assume resolution of singularities, while those of

2000 Mathematics Subject Classification. 14F42,19E15,19E20. 
[26] do. However, the paper takes its inspiration both from [9, 10] and [26].

This article is divided into three sections. The first one is technical and could be skipped at first reading: after recalling the main properties of motivic cohomology and adding a few complements in subsection 1.1, it "straightens out" the functoriality of Bloch's cycle complexes [1] in subsection 1.2, transforming them into presheaves of abelian groups on the category of essentially smooth schemes over a given base field: the original Bloch complexes are only contravariant up to homotopy. See theorem 1.17. Such a straightening is needed in the next section. We could have relied on Suslin's moving lemma in [25, th. 2.1], as suggested to the author by Suslin. Rather, we prefer to apply a more universal construction, and replace the Bloch complex by the homotopy inverse limit of its (quasi-isomorphic) subcomplexes with good position conditions for increasing collections of locally closed subsets. In subsection 1.3, we similarly "straighten-out" the GeisserLevine cycle class map of [10], and in subsection 1.4 we extend it to a motivic- $l$-adic cycle class map.

The second section is where we "revisit" the Geisser-Levine method. A useful device is the introduction of a new topology, well-adapted to the handling of multirelative cohomology groups: the open-closed topology, which plays a rôle rather similar to that of the cdh topology in [26] without forcing one to assume resolution of singularities. We also use some techniques from [6].

In Definition 2.14 we introduce the important notion of type at infinity of a complex of Zariski sheaves on $S m / k$, and in Definition 2.16 the related notion of malleability (roughly: the type at infinity is 0). The fundamental result of Geisser-Levine ([9, cor. 4.4], see also [26, Cor. 9.7]) may then be reformulated as follows: motivic cohomology is malleable in weights $>0$ (Theorem 2.28).

The main result of Section 2 is Theorem 2.34: a map from a version of motivic cohomology to a "cohomology theory" in a suitable sense is an isomorphism if and only if it is in weight 0 and the cohomology theory is malleable in weights $>0$. This can be viewed as a "uniqueness theorem" for motivic cohomology. In Corollary 2.38, we show how one can use it to recover the results of $[9,10]$.

In the third section, we apply the previous results to prove the equivalence between three conjectures (Theorem 3.4). The first one, Conjecture 3.1, is the conjunction of conjectures of Tate and Beilinson on algebraic cycles over a finite field. The second one, Conjecture 3.2, is an isomorphism between a version of motivic cohomology and continuous étale cohomology (in the sense of Jannsen). The third one, 
Conjecture 3.3, is that continuous étale cohomology with coefficients in $\mathbb{Q}_{l}(n)$ over $S m / \mathbb{F}_{p}$ is malleable for $n>0$. So we get an equivalence between a purely cycle-theoretic conjecture with rational coefficients (Conjecture 3.1) and a purely cohomological conjecture with rational coefficients (Conjecture 3.3), via a mixed conjecture with integral coefficients (Conjecture 3.2). The equivalence between Conjectures 3.1 and 3.2 had been given in the preprint [15] under resolution of singularities; here this assumption is dropped. It is our hope that Conjecture 3.1 will eventually be proven through this approach.

Consequences of the equivalence between Conjectures 3.1, 3.2 and 3.3 will be given elsewhere [17].

We fix a base field $k$. We denote by

- $S c h / k$ the category of separated $k$-schemes of finite type;

- $S m / k$ the full subcategory of $S c h / k$ consisting of smooth schemes.

We shall occasionally use the notations $\widetilde{S c h} / k$ and $\widetilde{S m} / k$ to denote the categories of schemes (resp. smooth schemes) essentially of finite type over $k$.

\section{FunCtORIALity OF MOTIVIC COHOMOLOGY}

1.1. Review of motivic cohomology. We shall define motivic cohomology groups (resp. Borel-Moore motivic homology groups) for a smooth quasi-projective $k$-variety (resp. for a quasi-projective $k$ variety) as Bloch's higher Chow groups [1] renumbered. (Later, we remove the quasiprojectiveness assumption.) Let us recall their definition:

1.1. Definition. Let $k$ be a field; denote by $\Delta^{\bullet}$ the standard cosimplicial scheme over $k$ (with $\Delta^{p}=\operatorname{Spec} k\left[t_{0}, \ldots, t_{p}\right] /\left(\sum t_{i}-1\right)$ ).

a) For $X$ a scheme essentially of finite type over $k$, the homological cycle complex of dimension $n$ of $X$ is the chain complex $z_{n}(X, *)$ associated to the simplicial abelian group $z_{n}(X, \bullet)$, where, for all $p, z_{n}(X, p)$ is the group of cycles of dimension $n+p$ on $X \times \Delta^{p}$ meeting all faces properly; the faces and degeneracies are induced by those of $\Delta^{\bullet}$. The weight $n$ Borel-Moore motivic complex of $X$ is the complex

$$
\mathcal{L}(X, n)=z_{n}(X, *)[2 n] .
$$

We denote by $L(X, n)$ its class in $D^{-}(A b)$, the derived category of bounded below chain complexes of abelian groups.

b) Suppose $X$ of pure dimension $d$. The cohomological cycle complex of codimension $n$ of $X$ is the cochain complex $z^{n}(X, *)$ deduced from the chain complex $z_{d-n}(X, *)$ (i.e. $\left.z^{n}(X, p)=z_{d-n}(X, p)\right)$. The weight 
$n$ motivic complex of $X$ is the complex

$$
\mathcal{Z}(X, n)=z^{n}(X, *)[-2 n]
$$

We denote by $\mathbb{Z}(X, n)$ its class in $D^{-}(A b)$. Therefore,

$$
L(X, n)=\mathbb{Z}(X, d-n)[2 d] .
$$

If $X$ is a disjoint union of pure dimensional schemes $X_{i}$, we set $\mathcal{Z}(X, n)=$ $\bigoplus \mathcal{Z}\left(X_{i}, n\right)$.

(We shall use b) mostly when $X$ is essentially smooth).

We shall usually consider $\mathcal{L}(X, n)$ as a chain complex and $\mathcal{Z}(X, n)$ as a cochain complex. Of course this is just a matter of notation, as one can pass from a chain complex $\left(C_{i}\right)$ to a cochain complex $\left(D^{i}\right)$ by setting $D^{i}=C_{-i}$.

Let us list the main properties of the complexes $\mathcal{L}(X, n)$ and $\mathcal{Z}(X, n)$ $[1,2,21,10]$. Property c) will be strengthened in subsection 1.2.

1.2. Theorem. a) [1, prop. 1.3] Elementary fonctoriality. The BorelMoore motivic complexes are covariant for proper maps and contravariant for flat, equidimensional maps.

b) $[1$, th. 4.1], [21, Part I, th. II.3.5.14] Smooth contravariance. The cohomology groups of $\mathcal{Z}(X, n)$ are contravariant for arbitrary maps between quasi-projective smooth schemes.

c) $[1$, th. 2.1] [21, Part I, prop. II.3.6.2] Homotopy invariance. Let $X \in S m / k$. Then, for all $n, q$,

$$
H^{q}(\mathbb{Z}(X, n)) \stackrel{f^{*}}{\longrightarrow} H^{q}\left(\mathbb{Z}\left(X \times \mathbf{A}^{1}, n\right)\right)
$$

is an isomorphism, where $f: X \times \mathbf{A}^{1} \rightarrow X$ is the first projection. For $X \in S c h / k$, the maps

$$
H_{q}(L(X, n)) \rightarrow H_{q+2}\left(L\left(X \times \mathbf{A}^{1}, n+1\right)\right)
$$

are isomorphisms as well.

d) $[1, \S 5],[10, \S 8]$ Products. For any smooth $X$, there are homotopy commutative and associative products

$$
\mathbb{Z}(X, m) \stackrel{L}{\otimes} \mathbb{Z}(X, n) \rightarrow \mathbb{Z}(X, m+n) .
$$

e) [1, th. 3.1], [2] Localisation. Let $i: Z \rightarrow X$ be a closed immersion of $k$-schemes of finite type, with $X$ quasi-projective, and let $j: U \rightarrow X$ be the corresponding open immersion. Then, for all $n$, the sequence of complexes

$$
\mathcal{L}(Z, n) \stackrel{i_{*}}{\longrightarrow} \mathcal{L}(X, n) \stackrel{j^{*}}{\longrightarrow} \mathcal{L}(U, n)
$$


defines an exact triangle in $D^{-}(A b)$. In particular, if $X=U \cup V$, with $U, V$ open, then the sequence of complexes

$$
\mathcal{L}(X, n) \stackrel{\left(j_{j_{V}}\right)}{\longrightarrow} \mathcal{L}(U, n) \oplus \mathcal{L}(V, n) \stackrel{\left(j_{U}^{\prime},-j_{V}^{\prime}\right)}{\longrightarrow} \mathcal{L}(U \cap V, n)
$$

defines an exact triangle (Mayer-Vietoris), where $j_{U}, j_{V}, j_{U}^{\prime}, j_{V}^{\prime}$ are the relevant open immersions.

By a), for any $X \in S c h / k, U \mapsto \mathcal{L}(U, n)$ determines in particular a (cochain) complex of presheaves over the small Zariski site of $X$ : we denote the associated complex of sheaves by $\mathcal{L}(n)_{X}$, and its class in the derived category by $L(n)_{X}$. For $X$ smooth, we denote similarly by $\mathcal{Z}(n)_{X}$ the complex of Zariski sheaves associated to $U \mapsto \mathcal{Z}(U, n)$, and by $\mathbb{Z}(n)_{X}$ its class in the derived category. We then have the following supplementary properties.

1.3. Theorem. f) [1, th. 3.2] Local-global principle. For all quasiprojective $X \in S c h / k$ and all $n \in \mathbb{Z}$, the augmentation

$$
\mathcal{L}(X, n) \rightarrow \mathbb{H}_{\text {Zar }}\left(X, L(n)_{X}\right)
$$

is a quasi-isomorphism, where the right hand side denotes the total complex associated to the Godement resolution of $L(n)_{X}$. In particular, if $X$ is smooth quasi-projective, the augmentation

$$
\mathcal{Z}(X, n) \rightarrow \mathbb{H}_{\text {Zar }}\left(X, \mathbb{Z}(n)_{X}\right)
$$

is an isomorphism for all $n \geq 0$.

g) $[1$, th. 10.1] Gersten's conjecture. Let $X \in S m / k$. For all $n, q$, denote by $\mathcal{H}^{q}(\mathbb{Z}(n))$ the Zariski sheaf on $X$ associated to $U \mapsto H^{q}(\mathbb{Z}(U, n))$. Then there are Gersten resolutions:

$$
\begin{aligned}
0 \rightarrow \mathcal{H}^{q}(\mathbb{Z}(n)) \rightarrow \coprod_{x \in X^{(0)}}\left(i_{x}\right)_{*} H^{q}(\mathbb{Z}(k(x), n)) & \\
& \rightarrow \coprod_{x \in X^{(1)}}\left(i_{x}\right)_{*} H^{q-1}(\mathbb{Z}(k(x), n-1)) \rightarrow \ldots
\end{aligned}
$$

where $X^{(p)}$ denotes the set of points of $X$ of codimension $p$.

h) $[1$, th. 9.1], [20, th. 3.1] Relationship with algebraic $K$-theory. There are isomorphisms for $X$ smooth quasi-projective over a field

$$
H^{p}(\mathbb{Z}(X, n)) \otimes \mathbb{Q} \simeq g r^{n} K_{2 n-p}(X)_{\mathbb{Q}}
$$

where $K_{*}(X)$ denotes Quillen's $K$-theory of $X$ and $g r^{n}$ refers to the gamma-filtration [24]. 
Theorem $1.3 \mathrm{f}$ ) implies that, for $X$ quasi-projective, there are isomorphisms for all $n, q$

$$
\begin{gathered}
H^{q}(L(X, n)) \stackrel{\sim}{\longrightarrow} \mathbb{H}_{\text {Zar }}^{q}\left(X, L(n)_{X}\right) \\
H^{q}(\mathbb{Z}(X, n)) \stackrel{\sim}{\longrightarrow} \mathbb{H}_{\text {Zar }}^{q}\left(X, \mathbb{Z}(n)_{X}\right) \quad(X \text { smooth }) .
\end{gathered}
$$

For $X$ arbitrary, we shall denote the right hand side of these isomorphisms respectively by $H_{-q}^{c}(X, \mathbb{Z}(n))$ and $H^{q}(X, \mathbb{Z}(n))$.

\subsection{Remarks.}

(1) In fact, theorem $1.3 \mathrm{~g}$ ) follows formally from theorem $1.2 \mathrm{c}$ ) and e), by results of [6]. Indeed, one checks easily by Noetherian induction that localisation implies étale excision for closed subsets of smooth schemes (compare proof of theorem 2.24).

(2) If $\left(X_{i}\right)_{i \in I}$ is a projective system of locally equidimensional schemes with flat transition morphisms, and if $X=\lim X_{i}$, then for all $n$ one has $z^{n}(X, *)=\lim _{\longrightarrow} z^{n}\left(X_{i}, *\right)$. In particular, if $X$ is integral with function field $K$, then $\lim _{U} z^{n}(U, *)$ $=z^{n}(K, *)$, where the limit is over nonempty open subsets of $X$. This is used implicitely in theorem $1.3 \mathrm{~g}$ ) and also below in the proof of proposition 1.7, (1.2) and (1.3). The similar statement is obviously wrong with homological cycles, even if the relative dimensions are 0 , as Krull dimension is not continuous.

Here is a reformulation of theorem $1.2 \mathrm{c}$ ) and e) in derived terms:

1.5. Proposition. a) For all $X \in S m / k$ and all $n \geq 0$, the morphism $\mathbb{Z}(n)_{X} \rightarrow R f_{*} \mathbb{Z}(n)_{\mathbf{A}^{1} \times X}$ is an isomorphism, where $f: \mathbf{A}^{1} \times X \rightarrow X$ is the first projection. For $X \in S c h / k$, the similar map

$$
L(n)_{X} \rightarrow R f_{*} L(n+1)_{\mathbf{A}^{1} \times X}[-2]
$$

is an isomorphism.

b) In the situation of theorem 1.2 e), the direct image map $i_{*}$ induces an isomorphism

$$
L(n)_{Z} \stackrel{\sim}{\longrightarrow} R i^{!} L(n)_{X}
$$

for all $n \in \mathbb{Z}$. In particular, if $i: Z \rightarrow X$ is a smooth $k$-pair of codimension $c$, then $i_{*}$ induces for all $n \geq 0$ an isomorphism

$$
\mathbb{Z}(n)_{Z} \stackrel{\sim}{\longrightarrow} R i ! \mathbb{Z}(n+c)_{X}[2 c]
$$

Proof. a) follows from theorem 1.2 c) and theorem $1.3 \mathrm{f}$ ), and b) follows from theorem $1.2 \mathrm{e}$ ) and theorem $1.3 \mathrm{f}$ ).

The following proposition gives a computation of some motivic cohomology groups: 
1.6. Proposition. Let $X$ be an essentially smooth scheme over $k$. Then,

(i) $H^{p}(X, \mathbb{Z}(n))=0$ for $p>2 n$.

(ii) $H^{2 n}(X, \mathbb{Z}(n))$ is canonically isomorphic to the $n$-th Chow group $C H^{n}(X)$.

Moreover

(iii) $\mathcal{H}^{p}\left(\mathbb{Z}(n)_{X}\right)=0$ for $p>n$.

(iv) $\mathcal{H}^{n}\left(\mathbb{Z}(n)_{X}\right) \simeq \widetilde{K}_{n}^{M}$, where $\widetilde{K}_{n}^{M}(X)$ is the $n$-th unramified Milnor $K$-sheaf of $X$, i.e.

$$
\widetilde{K}_{n}^{M}=\operatorname{Ker}\left(\bigoplus_{x \in X^{(0)}}\left(i_{x}\right)_{*} K_{n}^{M}(k(x)) \rightarrow \bigoplus_{x \in X^{(1)}}\left(i_{x}\right)_{*} K_{n-1}^{M}(k(x))\right)
$$

where $X^{(p)}$ denotes the set of points of codimension $p$ in $X$ and $i_{x}$ is the inclusion of a point $x$ in $X$.

Proof. (i) and (ii) are obvious from the definition of the motivic complex. For (iii) and (iv), we reduce to the case of a field by theorem $1.3 \mathrm{~g}$ ). In this case, (iii) is obvious and (iv) is the Nesterenko-Suslin theorem [23].

The next proposition generalises the previous one to the case of singular schemes. For a chain complex $C$. and an integer $n$, we denote by $\tau^{\leq n} C$. the truncation of $C$. such that

$$
H_{q}\left({ }^{\leq n} C .\right)= \begin{cases}H_{q}(C .) & \text { for } q \leq n \\ 0 & \text { else }\end{cases}
$$

so that $\tau^{\leq n} C$. $=\tau_{\geq-n} C$, where $C$ is the associated cochain complex.

1.7. Proposition. Let $X \in S c h / k$, and $d=\operatorname{dim} X$. Then

$$
\tau^{\leq n+d} L(n)_{X} \simeq M(-n)_{X}[n]
$$

where $M(n)_{X}$ denotes the (homological) Gersten complex of sheaves of Kato [18]

$$
\cdots \rightarrow \bigoplus_{x \in X_{(1)}}\left(i_{x}\right)_{*} K_{n+1}^{M}(k(x)) \rightarrow \bigoplus_{x \in X_{(0)}}\left(i_{x}\right)_{*} K_{n}^{M}(k(x)) \rightarrow 0
$$

in which $X_{(p)}$ denotes the set of points of dimension $p$ in $X$ and the last term of the complex is in homological degree 0.

Proof. Generally, let $C_{(0)} \rightarrow C_{(1)} \rightarrow \cdots \rightarrow C_{(d)}=C$ be a sequence of (chain) complexes in some abelian category. Define $C_{(p / p-1)}$ as the cone of the morphism $C_{(p-1)} \rightarrow C_{(p)}$. We have a double complex

$$
C_{. .}: C_{(d / d-1)} \rightarrow C_{(d-1 / d-2)}[1] \rightarrow \cdots \rightarrow C_{(1 / 0)}[d-1] \rightarrow C_{(0)}[d] \rightarrow 0
$$


and the map $C \rightarrow C_{(d / d-1)}$ defines a homotopy equivalence $C \stackrel{\sim}{\longrightarrow}$ $\operatorname{Tot}(C .$.$) .$

In our case, consider the niveau filtration on Borel-Moore motivic homology:

$$
F_{p} z_{n}(X, *)=\underset{\operatorname{dim} Z \leq p}{\lim _{n}} z_{n}(Z, *)
$$

where the direct limit runs through reduced closed subschemes of $X$ (note that this does not obviously coincide with the definition in $[1$, $\S 10]$ when $X$ is smooth).

Sheafifying this for the Zariski topology gives a quasi-isomorphism

$\mathcal{L}(n)_{X} \stackrel{\sim}{\longrightarrow} \operatorname{Tot}\left(\mathcal{L}_{(d / d-1)}(n)_{X} \rightarrow \mathcal{L}_{(d-1 / d-2)}(n)_{X}[1] \rightarrow \cdots \rightarrow \mathcal{L}_{(0)}(n)_{X}[d]\right)$

where $\mathcal{L}_{(p / p-1)}(n)_{X}$ denotes the cone of the sheafification of $F_{p-1} z_{n}(-, *)$ $\rightarrow F_{p} z_{n}(-, *)$.

By theorem $1.2 \mathrm{e}), \mathcal{L}_{(p / p-1)}(n)_{X}$ is quasi-isomorphic to

$$
\bigoplus_{x \in X_{(p)}}\left(i_{x}\right)_{*} \mathcal{Z}(k(x), p-n)[2 p]
$$

so that (1.1) reads

$$
\begin{aligned}
& \mathcal{L}(n)_{X} \stackrel{\sim}{\longrightarrow} \operatorname{Tot}\left(\bigoplus_{x \in X_{(d)}}\left(i_{x}\right)_{*} \mathcal{Z}(k(x), d-n)[2 d] \rightarrow \cdots \rightarrow\right. \\
& \left.\bigoplus_{x \in X_{(p)}}\left(i_{x}\right)_{*} \mathcal{Z}(k(x), p-n)[p+d] \rightarrow \cdots \rightarrow \bigoplus_{x \in X_{(0)}}\left(i_{x}\right)_{*} \mathcal{Z}(k(x),-n)[d]\right) .
\end{aligned}
$$

Noting that $\mathcal{Z}(k(x), p-n)$ is acyclic in (homological) degrees $<n-p$, it follows that $\tau^{\leq n+d} L(n)_{X}$ is quasi-isomorphic to the Gersten complex

$$
\bigoplus_{x \in X_{(d)}}\left(i_{x}\right)_{*} H^{d-n}(k(x), \mathbb{Z}(d-n)) \rightarrow \cdots \rightarrow \bigoplus_{x \in X_{(0)}}\left(i_{x}\right)_{*} H^{-n}(k(x), \mathbb{Z}(-n)) .
$$

As seen above, the $p$-th term of this complex is isomorphic to $\bigoplus_{x \in X_{(p)}}\left(i_{x}\right)_{*} K_{p-n}^{M}(k(x))$; moreover, by (the proof of) [9, lemma 3.2], the differentials coincide with those from [18].

For any $X \in S c h / k$, the niveau filtration used in the proof of proposition 1.7 determines by theorem 1.2 e) the niveau spectral sequence

$$
E_{p, q}^{1}=\coprod_{x \in X_{(p)}} H^{p-q}(k(x), \mathbb{Z}(p-n)) \Rightarrow H_{p+q}^{c}(X, \mathbb{Z}(n)) .
$$


Similarly, when $X$ is smooth, we have the coniveau filtration on motivic cohomology, yielding the coniveau spectral sequence:

$$
E_{1}^{p, q}=\coprod_{x \in X^{(p)}} H^{q-p}(k(x), \mathbb{Z}(n-p)) \Rightarrow H^{p+q}(X, \mathbb{Z}(n)) .
$$

Theorem $1.3 \mathrm{~g})$ implies that the $E_{2}$-terms of (1.3) are given by $E_{2}^{p, q}=$ $H_{\text {Zar }}^{p}\left(X, \mathcal{H}^{q}(\mathbb{Z}(n))\right.$.

1.2. A global motivic complex. We want to strengthen theorem $1.2 \mathrm{~b})$ to make $X \mapsto \mathbb{Z}(X, n)$ an object of $D^{-}\left(A b\left((S m / k)_{\text {Zar }}\right)\right)$, the derived category of Zariski sheaves over the big smooth Zariski site of Spec $k$. This is not straightforward, as the motivic complexes are not contravariant on the nose.

1.8. Definition. Let $E$ be a subcategory of $S c h / k$ stable under open immersions. Let $\mathcal{C}$ be a contravariant functor from $E$ to $C(A b)$, the category of complexes of abelian groups. We say that $\mathcal{C}$ satisfies MayerVietoris if, for any triple $(X, U, V)$ where $X=U \cup V, U, V$ are open in $X$ and $X \in E$, the sequence

$$
\mathcal{C}(X) \rightarrow \mathcal{C}(U) \oplus \mathcal{C}(V) \rightarrow \mathcal{C}(U \cap V)
$$

defines an exact triangle in $D(A b)$, the derived category of the category of abelian groups.

With notation as in definition 1.8, let $C(A b)^{E^{o}}=C\left(A b^{E^{o}}\right)$ be the category of contravariant functors from $E$ to $C(A b)$, and let $C(A b)_{M V}^{E^{o}}$ be the full subcategory of functors satisfying Mayer-Vietoris. Let $E_{\text {aff }}$ be the full subcategory of $E$ consisting of affine schemes in $E$. We have a naturally commutative diagram of categories and functors:

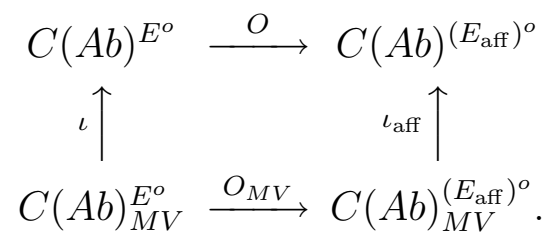

1.9. Proposition. There exists a functor $\check{\mathbb{H}}_{\text {aff }}^{\cdot}: C(A b)^{\left(E_{\text {aff }}\right)^{o}} \rightarrow$ $C(A b)_{M V}^{E^{o}}$ and a natural transformation $\varepsilon: I d \rightarrow \iota_{\text {aff }} O_{M V} \check{\mathbb{H}}_{\text {aff }}$ such that:

(i) $\check{\mathbb{H}}_{\text {aff }}^{\cdot}$ transforms quasi-isomorphisms into quasi-isomorphisms.

(ii) For $\mathcal{C} \in C(A b)^{\left(E_{\text {aff }}\right)^{o}}, \mathcal{C} \in C(A b)_{M V}^{\left(E_{\text {aff }}\right)^{o}}$ if and only if $\varepsilon_{\mathcal{C}}$ is a quasi-isomorphism.

Proof. Let $\mathcal{C}:\left(E_{\text {aff }}\right)^{o} \rightarrow C(A b)$ be a functor. Let $X \in E$ and let $\mathcal{U}=\left(U_{i}\right)_{i \in I}$ be a finite open cover of $X$ by affine schemes. Since $X$ is 
separated, all intersections of $U_{i}$ are affine. This allows us to consider the Cech double complex:

$$
\bigoplus_{i \in I} \mathcal{C}\left(U_{i}\right) \rightarrow \bigoplus_{(i, j) \in I \times I} \mathcal{C}\left(U_{i} \cap U_{j}\right) \rightarrow \ldots
$$

Let $\check{\mathbb{H}} \cdot(\mathcal{U}, \mathcal{C})$ be the total complex of this double complex. We define

$$
\check{\mathbb{H}}_{\text {aff }}(X, \mathcal{C})=\underset{\mathcal{U}}{\lim } \check{\mathbb{H}} \cdot(\mathcal{U}, \mathcal{C})
$$

Let $f: Y \rightarrow X$ be a morphism in $E$, and let $\mathcal{U}=\left(U_{i}\right)_{i \in I}$ be a finite open cover of $X$ by affine schemes. Then $\left(f^{-1}\left(U_{i}\right)\right)_{i \in I}$ is a finite open cover of $Y$ by not necessarily affine schemes. Choosing for all $i$ a finite open cover $\left(V_{i j}\right)_{j \in J_{i}}$ of $f^{-1}\left(U_{i}\right)$ by affine schemes, and letting $\mathcal{V}=\left(V_{i j}\right)$, we get a morphism

$$
\check{\mathbb{H}} \cdot(\mathcal{U}, \mathcal{C}) \rightarrow \check{\mathbb{H}}^{\cdot}(\mathcal{V}, \mathcal{C})
$$

Passing to the limit, we get a morphism $f^{*}: \check{\mathscr{H}}_{\text {aff }}^{\cdot}(X, \mathcal{C}) \rightarrow \check{\mathbb{H}}_{\text {aff }}(Y, \mathcal{C})$. Hence $\check{\mathbb{H}}_{\text {aff }}^{\cdot}(X, \mathcal{C})$ is contravariant in $X$ (check that this construction respects composition). We denote this functor by $\check{\mathbb{H}}_{\text {aff }}(\mathcal{C})$. Clearly, $\mathcal{C} \mapsto \check{\mathbb{H}}_{\text {aff }}^{\cdot}(\mathcal{C})$ defines a functor

$$
\check{\mathbb{H}}_{\text {aff }}: C(A b)^{\left(E_{\text {aff }}\right)^{o}} \rightarrow C(A b)^{E^{o}} .
$$

1.10. Lemma. a) For all $\mathcal{C}, \check{\mathscr{H}}_{\text {aff }}^{\cdot}(\mathcal{C})$ satisfies Mayer-Vietoris in E.

b) Let $\mathcal{C}, \mathcal{D} \in C(A b)^{\left(E_{\text {aff }}\right)^{\circ}}$ and $\varphi: \mathcal{C} \rightarrow \mathcal{D}$ be a morphism such that, for all $X, \varphi_{X}$ is a quasi-isomorphism (briefly: $\varphi$ is a quasi-isomorphism). Then $\check{\mathbb{H}}_{\text {aff }}(\varphi)$ is also a quasi-isomorphism.

Indeed, let $X=U \cup V$, where $U, V$ are open. Let $\mathcal{U}=\left(U_{i}\right)_{i \in I}$ be a finite open affine cover of $U$ and $\mathcal{V}=\left(V_{j}\right)_{j \in J}$ a finite open affine cover of $V$. Then $\mathcal{U} \cup \mathcal{V}$ is a finite open affine cover of $X$, while $\mathcal{U} \cdot \mathcal{V}=$ $\left(U_{i} \cap V_{j}\right)_{(i, j) \in I \times J}$ is a finite open affine cover of $U \cap V$. One sees easily, by induction on $|I|$ and $|J|$, that the sequence

$$
\check{\mathbb{H}} \cdot(\mathcal{U} \cup \mathcal{V}, \mathcal{C}) \rightarrow \check{\mathbb{H}}^{\cdot}(\mathcal{U}, \mathcal{C}) \oplus \check{\mathbb{H}}^{\cdot}(\mathcal{V}, \mathcal{C}) \rightarrow \check{\mathbb{H}}^{\prime}(\mathcal{U} \cdot \mathcal{V}, \mathcal{C})
$$

defines an exact triangle in the derived category. Since covers of the form $\mathcal{U} \cup \mathcal{V}$ are cofinal among finite open affine covers of $X$ and covers of the form $\mathcal{U} \cdot \mathcal{V}$ are cofinal among finite open affine covers of $U \cap V$, this proves a), and b) follows readily from the Cech spectral sequence for a finite affine cover and a passage to the limit.

We have checked condition (i) of proposition 1.9. Let $X \in E_{\text {aff }}$. There is an obvious augmentation map:

$$
\mathcal{C}(X) \rightarrow \check{\mathbb{H}}_{\text {aff }}(X, \mathcal{C})
$$


which defines the natural transformation $\varepsilon$ of proposition 1.9. The Mayer-Vietoris condition being invariant under quasi-isomorphisms, sufficiency in condition (ii) is clear, and necessity is proved by passage to the limit from the case of a finite covering, the latter being easily proved by induction on the number of terms of the covering.

1.11. Proposition. Let Smaff/ $k$ be the full subcategory of $S m / k$ consisting of smooth, affine varieties, and let $\mathcal{C}:(\text { Smaf } f / k)^{\circ} \rightarrow C(A b)$ be a functor as in definition 1.8, satisfying Mayer-Vietoris. Then there exists an object $C \in D\left(A b\left((S m / k)_{\mathrm{Zar}}\right)\right)$ such that, for all $X \in S m a f f / k$, the class of $\mathcal{C}(X)$ in $D(A b)$ is isomorphic to $R \Gamma(X, C)$.

Proof. Let $\mathcal{D}=\check{\mathbb{H}}_{\text {aff }}(\mathcal{C})$, where $\check{\mathbb{H}}_{\text {aff }}$ is as in proposition 1.9. For $X \in$ $S m / k$, let $\mathbb{H}_{\text {Zar }}(X, \mathcal{D})$ be the total complex of the Godement resolution of the sheaf of complexes associated to $\mathcal{D}$. Since $\mathcal{D}$ satisfies MayerVietoris, the argument of [29, ex. 2.5] shows that the augmentation

$$
\mathcal{D}(X) \rightarrow \mathbb{H}_{\text {Zar }}(X, \mathcal{D})
$$

is a quasi-isomorphism. Therefore, when $X$ is affine, the composition

$$
\mathcal{C}(X) \rightarrow \mathcal{D}(X) \rightarrow \mathbb{H}_{\text {Zar }}(X, \mathcal{D})
$$

is a quasi-isomorphism. We may therefore take for $C$ the class of the complex of sheaves associated to $\mathcal{D}$.

We are now going to produce out of the $z^{n}(X, *)$ a functor $\hat{z}^{n}$ verifying the hypotheses of Proposition 1.11. The following lemma is obviously not in its most general form, but it will suffice for our purpose.

1.12. Lemma. Let $\mathcal{C}$ be a category and $I$ a covariant functor from $\mathcal{C}$ to the category of ordered sets. For each $X \in \mathcal{C}$, let $\left(C_{i}(X)\right)_{i \in I(X)}$ be an inverse system of chain complexes of abelian groups. Assume given, for any morphism $f: Y \rightarrow X$ in $\mathcal{C}$ and any $i \in I(Y)$, a chain homomorphism $f_{i}^{*}: C_{f(i)}(X) \rightarrow C_{i}(Y)$ (of degree 0 ) with the following properties:

(1) If $i \leq j \in I(Y)$, then the diagram

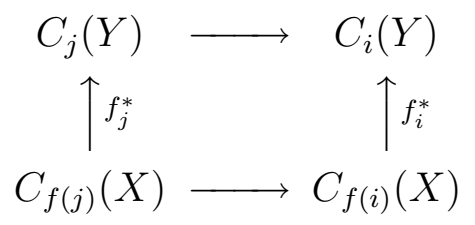

commutes. 
(2) If $g: Z \rightarrow Y$ is another morphism and $i \in I(Z)$, then the diagram

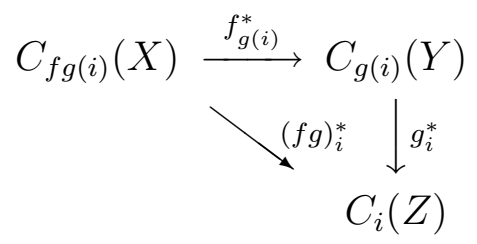

commutes.

Then the above assignment defines a functor $\hat{C}: \mathcal{C}^{o} \rightarrow C(A b)$ such that

$$
\hat{C}(X)=\operatorname{holim}_{i \in I(X)} C_{i}(X)
$$

for any $X \in \mathcal{C}$.

(See $[3$, ch. XI] for a definition of the homotopy limit functor.)

Proof. For $f: Y \rightarrow X$ a morphism in $\mathcal{C}$, define $f^{*}: \hat{C}(X) \rightarrow \hat{C}(Y)$ as the composition

$$
\operatorname{holim}_{i \in I(X)} C_{i}(X) \rightarrow \operatorname{holim}_{j \in I(Y)} C_{f(i)}(X) \rightarrow \operatorname{holim}_{j \in I(Y)} C_{i}(Y)
$$

where the first map is given by restriction of the inverse system and the second one is induced by the $f_{i}^{*}$, thanks to Condition (1). Then Condition (2) ensures that, given a second map $g: Z \rightarrow Y$, one has $(f g)^{*}=g^{*} f^{*}$.

1.13. Definition. Let $X \in S m a f f / k$ and $n>0$. We denote by $I^{n}(X)$ the set of finite collections $\left(Z_{\alpha}, n_{\alpha}\right)_{\alpha \in A}$, where the $Z_{\alpha}$ are locally closed irreducible subsets of $X$ and the $n_{\alpha}$ are integers $\leq n$. We put on $I^{n}(X)$ the following order: $\left(Z_{\alpha}, n_{\alpha}\right)_{\alpha \in A} \leq\left(T_{\beta}, m_{\beta}\right)_{\beta \in B}$ if there exists an injection $\varphi: A \hookrightarrow B$ such that $T_{\varphi(\alpha)}=Z_{\alpha}$ and $m_{\varphi(\alpha)} \geq n_{\alpha}$.

1.14. Remark. The ordered set $I^{n}(X)$ is actually filtering (any two elements have an upper bound).

For $i=\left(Z_{\alpha}, n_{\alpha}\right)_{\alpha \in A} \in I^{n}(X)$, recall from [21, Part I, Lemma II.3.5.1] the subcomplex $z_{i}^{n}(X, *)$ of $z^{n}(X, *)$ : its $p$-th term is the free abelian group on integral subschemes $Z$ of codimension $n$ in $X \times \Delta^{p}$ such that $Z$ meets all subschemes of the form $Z_{\alpha} \times F$ in codimension $\geq n_{\alpha}$, where $F$ is a face of $\Delta^{p}$.

More generally, let $X_{q} \stackrel{f_{q}}{\longrightarrow} \ldots \stackrel{f_{1}}{\longrightarrow} X_{0}$ be a chain of morphisms in Smaff $/ k$ and $i \in I^{n}\left(X_{q}\right)$. We define recursively $z_{f_{1}, \ldots, f_{q}, i}^{n}\left(X_{0}, *\right)$ as the subcomplex of $z^{n}(X, *)$ freely generated by those closed integral subschemes $Z$ of codimension $n$ such that $f_{1}^{*} Z$ is defined and, with 
obvious notation, $f_{1}^{*} Z \in z_{f_{2}, \ldots, f_{q}, i}^{n}\left(X_{1}, *\right)$ (for $q=0, z_{f_{1}, \ldots, f_{q}, i}^{n}\left(X_{0}, *\right):=$ $\left.z_{i}^{n}\left(X_{0}, *\right)\right)$. We have the following trivial observation:

$$
z_{f_{1}, \ldots, f_{q}, i}^{n}\left(X_{0}, *\right)=z_{f_{1} \circ \cdots \circ f_{q}, i}^{n}\left(X_{0}, *\right) .
$$

We shall need the following slight generalisation of [21, Part I, Lemma II.3.5.2]:

1.15. Lemma. Let $f: Y \rightarrow X$ a morphism in Smaff $/ k, n \geq 1$ and $i \in I^{n}(Y)$. Then there exists $j \in I^{n}(X)$ such that $z_{f, i}^{n}(X, *)=z_{j}^{n}(X, *)$.

Proof. We proceed as in the proof of the quoted lemma. For simplicity, we'll assume that $Y$ is connected (the general case is similar but notationally more painful). Let $i=\left(Z_{\alpha}, n_{\alpha}\right)_{\alpha \in A}, d=\operatorname{dim} Y$ and $d_{\alpha}=\operatorname{dim} Z_{\alpha}$. For $t \leq d$, let (as in loc. cit. ) $C_{t}$ be the subset of $X$ defined as the set of points $x$ such that each irreducible component of $f^{-1}(\overline{\{x\}})$ of maximal dimension has dimension $t$ over $k(x)$. Moreover, for each $\alpha \in A$, let $C_{t, \alpha}$ be the subset of $C_{t}$ consisting of those $x$ such that $f^{-1}(x) \subseteq Z_{\alpha}$. Then the $C_{t}$ and $C_{t, \alpha}$ are constructible subsets of $X$ and form filtrations respectively of $f(Y)$ and $f\left(Z_{\alpha}\right)$.

Write each $C_{t}$ (resp. $C_{t, \alpha}$ as a finite union of irreducible subsets $C_{t}^{l}$ (resp. $C_{t, \alpha}^{l}$, each of which is locally closed in $X$, and let $d_{t}^{l}=\operatorname{dim} C_{t}^{l}$ (resp. $d_{t, \alpha}^{l}=\operatorname{dim} C_{t, \alpha}^{l}$ ), so that we have

$$
\begin{gathered}
d_{t}^{l}+t \leq d \\
d_{t, \alpha}^{l}+t \leq d_{\alpha}
\end{gathered}
$$

for all $l, \alpha$.

If we now define

$$
\begin{gathered}
n_{t}^{l}=t+d_{t}^{l}-d+n \\
n_{t, \alpha}^{l}=t+d_{t, \alpha}^{l}-d_{\alpha}+n,
\end{gathered}
$$

the same dimension computation as in loc. cit. shows that $j=$ $\left(C_{t}^{l}, n_{t}^{l} ; C_{t, \alpha}^{l}, n_{t, \alpha}^{l}\right)$ verifies the conclusion of Lemma 1.15.

1.16. Definition. We denote by $\bar{I}^{n}(X)$ the set of those subcomplexes of $z^{n}(X)$ of the form $z_{i}^{n}(X, *)$, with $i \in I^{n}(X)$; we put on $\bar{I}^{n}(X)$ the opposite order to inclusion.

Clearly the natural map $I^{n}(X) \rightarrow \bar{I}^{n}(X)$ is surjective and nondecreasing; the set $\bar{I}^{n}(X)$ is still filtering. For $f: Y \rightarrow X$ a morphism in Smaff/k and $n \geq 1$, Lemma 1.15 defines a map $f: \bar{I}^{n}(Y) \rightarrow$ $\bar{I}^{n}(X)$, which is clearly non-decreasing. Formula (1.4) shows in one 
gulp that this makes $\bar{I}^{n}(X)$ a covariant functor in $X$ and that, setting $C_{i}(X)=z_{i}^{n}(X, *)$, the conditions of Lemma 1.12 are satisfied with $I(X)=\bar{I}^{n}(X)$.

1.17. Theorem. Let $n \geq 0$. There exists an object $\mathbb{Z}(n)$ in $D^{-}\left(A b\left((S m / k)_{\mathrm{Zar}}\right)\right)$ such that, for all quasi-projective $X \in S m / k$, one has $\mathbb{Z}(X, n) \simeq R \Gamma\left(X_{\text {Zar }}, \mathbb{Z}(n)\right)$ in $D(A b)$.

Proof. Define, for $X \in S m a f f / k$

$$
\hat{z}^{n}(X, *)=\operatorname{holim}_{i \in I^{n}(X)} z_{i}^{n}(X, *)=\operatorname{holim}_{i \in \bar{I}^{n}(X)} z_{i}^{n}(X, *) .
$$

Lemmas 1.12 and 1.15 show that $X \mapsto \hat{z}^{n}(X, *)$ defines a contravariant functor on smooth affine schemes. We now use the following moving lemma of Bloch and Levine [21, th. II.3.5.14]: for all $X \in S m a f f / k$ and all $i \in I^{n}(X)$, the inclusion

$$
z_{i}^{n}(X, *) \rightarrow z^{n}(X, *)
$$

is a quasi-isomorphism. Therefore the natural map $\hat{z}^{n}(X, *) \rightarrow z^{n}(X, *)$ is also a quasi-isomorphism; moreover, by theorem $1.2 \mathrm{e}$ ), the functor $\hat{z}^{n}$ verifies Mayer-Vietoris. Applying proposition 1.11, we find that there exists an object $\mathbb{Z}(n)$ of $D\left(A b\left((S m / k)_{\text {Zar }}\right)\right)$ satisfying the condition of theorem 1.17 for $X$ affine. But this extends to $X \in S m / k$ quasiprojective, by covering it by affine open sets and applying theorem 1.2 e) again.

1.18. Proposition. The natural map $\mathbb{Q}(n) \rightarrow R \alpha_{*} \alpha^{*} \mathbb{Q}(n)$ is an isomorphism in $D(A b(S m / k)$ ét $)$ for all $n \geq 0$.

Proof. It is enough to prove this on the small Zariski site of any $X \in S m / k$. In case $X=\operatorname{Spec} k$, we look at the hypercohomology spectral sequence

$$
E_{2}^{p, q}=H_{\text {ét }}^{p}\left(k, H^{q}(\bar{k}, \mathbb{Q}(n))\right) \Rightarrow H_{\text {ét }}^{p+q}(k, \mathbb{Q}(n)) .
$$

We have $E_{2}^{p, q}=0$ for $p>0$, because higher Galois cohomology groups are torsion. It remains to show that the map

$$
H^{p}(k, \mathbb{Q}(n)) \rightarrow H^{p}(\bar{k}, \mathbb{Q}(n))^{G_{k}}
$$

is an isomorphism for all $p$, where $G_{k}$ is the absolute Galois group of $k$. But this follows from the existence of transfers for étale morphisms in motivic cohomology [1, cor. 1.4].

The case where $X$ is local Artinian with residue field $k$ follows from the previous one, since obviously $z_{n}(X, *)=z_{n}(k, *)$. This extends to local Artinian $k$-algebras with another residue field. Finally, the general case follows from this one and theorem 1.2 e) by [29, prop. 2.8] (to apply loc. cit., either adapt the proof to the case of presheaves of 
complexes, or apply it directly to the presheaf of spectra associated to the presheaf of cycle complexes by the Dold-Kan construction).

We shall authorise ourselves of proposition 1.18 to drop in section 3 the index ét from the groups $H_{\text {ét }}^{i}(X, \mathbb{Q}(n))$, as they coincide with their Zariski couterparts.

1.19. Corollary. Proposition 1.5 holds after replacing $\mathbb{Z}(n)$ by $\alpha^{*} \mathbb{Z}(n)[1 / p]$, where $p$ is the exponential characteristic of $k$.

Proof. It is enough to check this after tensoring with $\mathbb{Q}$ and with $\mathbb{Z} / l$ for all $l \neq p$. The first case follows from proposition 1.18; the second one follows from theorem 1.22 and the corresponding properties of étale cohomology with finite coefficients [SGA4], [SGA41/2].

1.20. Remark. This corollary is already wrong for $n=0$ if one does not invert $p$ (étale cohomology with coefficients $\mathbb{Z} / p$ is not homotopy invariant in characteristic $p$ ).

1.3. The $(\bmod m)$ cycle map. For any abelian group $A$, we denote the object $\mathbb{Z}(n) \stackrel{L}{\otimes} A$ by $A(n)$. Let $\alpha$ denote the projection of the big étale site of Spec $k$ onto its big Zariski site, and for all $X \in S c h / k$, let $\alpha_{X}$ denote the restriction of $\alpha$ to the small étale site of $X$. We denote the group $\mathbb{H}^{q}\left(X_{\text {ét }}, \alpha_{X}^{*} \mathcal{Z}(n)_{X}\right)$ by $H_{\text {ét }}^{q}(X, \mathbb{Z}(n))$ (similarly with coefficients in $A$ ).

Let $m$ be an integer prime to char $k$ and $X \in S c h / k$. We are going to define morphisms

$$
\alpha^{*} \mathbb{Z} / m(n) \rightarrow \mu_{m}^{\otimes n}(n \geq 0)
$$

in $\mathcal{D}\left((S m / k)_{\text {ét }}\right)$. The issue is to globalise the construction of GeisserLevine ([10, §3.7], esp. Remark 3.9); this will strengthen loc. cit. , Prop. 4.2. As the method is exactly the same as in subsection 1.2, we'll only sketch the details.

For $i \in I^{n}(X)$, let $\mathcal{Z}_{i}^{n}(X, *)=z_{i}^{n}(X, *)[-2 n]$. In loc. cit. , Geisser and Levine construct the following chain of morphisms of complexes

$$
\begin{array}{cc}
\mathcal{Z}_{i}^{n}(X, *) \otimes \mathbb{Z} / m & \longrightarrow H^{2 n}\left(G_{(n)}^{*}\left(X \times \Delta^{*}, \mu_{m}^{\otimes n}\right)_{i}\right)[-2 n] \\
G^{*}\left(X \times \Delta^{*}, \mu_{m}^{\otimes n}\right) & \longleftarrow \\
\imath \uparrow & \tau_{\leq 2 n} G_{(n)}^{*}\left(X \times \Delta^{*}, \mu_{m}^{\otimes n}\right)_{i} \\
G^{*}\left(X, \mu_{m}^{\otimes n}\right) &
\end{array}
$$


where $G^{*}\left(-, \mu_{m}^{\otimes n}\right)$ denotes the complex of global sections of the Godement resolution of the étale sheaf $\mu_{m}^{\otimes n}, G_{(n)}^{*}\left(-, \mu_{m}^{\otimes n}\right)_{i}$ its subcomplex of classes with support in closed subsets of codimension $\geq n$ in good position (see loc. cit. for details) and $X \times \Delta^{*}$ is the standard cosimplicial scheme constructed out of the $\Delta^{q}$. Actually (1.5) is constructed in loc. cit. only for $i=\left(Z_{\alpha}, n_{\alpha}\right)$ of a certain type (the $Z_{\alpha}$ closed and $n_{\alpha}=n$ for all $\alpha$ ), but the general case is the same.

The vertical maps in (1.5) are isomorphisms by purity and homotopy invariance of the étale cohomology of $\mu_{m}^{\otimes n}$. The result is a collection of compatible maps in $D(A b)$ (loc. cit. , (3.9)):

$$
c l_{i}^{n}: \mathcal{Z}_{i}(X, *) \otimes \mathbb{Z} / m \rightarrow G^{*}\left(X, \mu_{m}^{\otimes q}\right) .
$$

The construction shows:

1.21. Lemma. a) The maps in diagram (1.5) commute with change of coefficients.

b) For $X, Y, f, i, j$ as in Lemma 1.15, the diagram of zigzags (1.5)

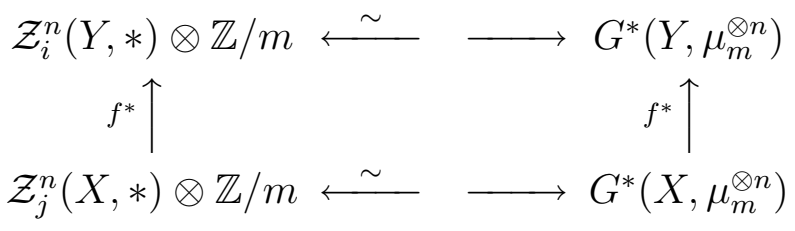

commutes.

c) (1.5) commutes with Gysin morphisms.

(Part c) of the lemma is [10, Lemma 4.4].)

The technique in the proof of theorem 1.17 now defines a morphism in $\mathcal{D}((S m / k)$ ét $)$

$$
\alpha^{*} \mathbb{Z} / m(n) \rightarrow \mu_{m}^{\otimes n}
$$

Using proposition 1.6, we see that the adjunction of this map yields a refined morphism

$$
\mathbb{Z} / m(n) \rightarrow \tau_{\leq n} R \alpha_{*} \mu_{m}^{\otimes n}
$$

which is studied in the next section.

1.22. Theorem. The morphism (1.6) is an isomorphism.

Proof. This is just a reformulation of [10, th. 1.5]. 
1.4. The $l$-adic cycle map. We fix a prime $l \neq$ char $k$ and introduce the objects

$$
\mathbb{Z}_{l}(n)^{c}:=R \lim _{\longleftarrow} \mu_{l^{\nu}}^{\otimes n} \in D^{+}\left((S m / k)_{\text {ét }}\right) .
$$

For any $n \in \mathbb{Z}, \mathbb{Z}_{l}(n)^{c}$ is a complex of ordinary étale sheaves; the reader should take care not to confuse it with the $l$-adic sheaf $\mathbb{Z}_{l}(n)$ of SGA 5. In fact, it is the image of $\mathbb{Z}_{l}(n)$ in $D^{+}((S m / k)$ ét $)$ by a suitable triangulated functor.

From Lemma 1.21, we deduce a morphism in the derived category, just as (1.6):

$$
\alpha^{*} \mathbb{Z}(n) \stackrel{L}{\otimes} \mathbb{Z}_{l} \rightarrow \mathbb{Z}_{l}(n)^{c} \quad(n \geq 0) .
$$

Let us examine an instance of this morphism. For any $X \in S m / k$, it induces homomorphisms

$$
H_{\text {ét }}^{p}(X, \mathbb{Z}(n)) \rightarrow H_{\text {cont }}^{p}\left(X, \mathbb{Z}_{l}(n)\right) .
$$

For $p=2 n$, by restriction to Zariski cohomology, we get a homomorphism

$$
C H^{n}(X)=H^{2 n}(X, \mathbb{Z}(n)) \rightarrow H_{\text {cont }}^{2 n}\left(X, \mathbb{Z}_{l}(n)\right) .
$$

1.23. Lemma. This homomorphism is the l-adic cycle class map of $[13,(6.14)]$. In particular, the composition

$$
C H^{n}(X) \rightarrow H_{\text {cont }}^{2 n}\left(X, \mathbb{Z}_{l}(n)\right) \rightarrow H_{\text {cont }}^{2 n}\left(\bar{X}, \mathbb{Z}_{l}(n)\right)^{G}
$$

is the classical l-adic cycle class map of Grothendieck et al. Here $G=$ $\operatorname{Gal}(\bar{k} / k)$ and $\bar{X}=X \otimes_{k} \bar{k}$, where $\bar{k}$ is a separable closure of $k$.

Proof. This follows easily from the fact that the isomorphism of theorem 1.22 is induced by a cycle class map for higher Chow groups extending the classical cycle map ( $c f .[10,3.7],[13,(3.23)])$.

\section{The Geisser-Levine method Revisited}

\subsection{The open-closed topology.}

2.1. Definition. Let $S$ be a scheme.

a) The big open-closed site on $S$ is the site $S_{\text {Oc }}$ given by the following Grothendieck pretopology:

- The objects of the underlying category are $S$-schemes; morphisms are all morphisms.

- Covers are generated by finite closed covers and finite open covers.

b) The small open-closed site on $S$ is the full subcategory $S_{\text {oc }} \subset S_{\text {Oc }}$ formed of reduced locally closed subschemes of $S$, provided with the open-closed covers. 
Note that an open-closed cover is in particular a cover by locally closed subsets, but the converse is not true in general. The openclosed topology is the coarsest topology which is finer than both the Zariski topology and the "irreducible" topology (given by finite closed covers).

By the usual yoga, there are adjoint morphisms of sites $\alpha^{*}: S_{\mathrm{oc}} \rightarrow$ $S_{\mathrm{Oc}}$ and $\alpha_{*}: S_{\mathrm{Oc}} \rightarrow S_{\mathrm{oc}}$ which induce inverse isomorphisms on sheaf cohomology: we shall call the latter open-closed cohomology and simply write it with an index oc. By definition, open-closed cohomology satisfies Mayer-Vietoris for open and for closed covers.

The following proposition gives some rules for computing in the openclosed topology (we refer for example to $[9,10]$ for multirelative cohomology groups.)

2.2. Proposition. a) Let $X$ be a scheme and $\left(Z_{i}\right)_{1 \leq i \leq k}$ a finite family of closed subsets of $X$. Then, for any complex of oc sheaves $C$, the natural map

$$
H_{\mathrm{oc}}^{*}\left(X, Z_{1}, \ldots, Z_{k}, C\right) \rightarrow H_{\mathrm{oc}}^{*}\left(X, Z_{1} \cup \cdots \cup Z_{k}, C\right)
$$

on relative cohomology groups is an isomorphism.

b) (Closed excision). Let $X=Z_{1} \cup Z_{2}$, with $Z_{1}, Z_{2}$ closed. Then for any coefficients $C$, the restriction map

$$
H_{\mathrm{oc}}^{*}\left(X, Z_{1}, C\right) \rightarrow H_{\mathrm{oc}}^{*}\left(Z_{2}, Z_{1} \cap Z_{2}, C\right)
$$

is an isomorphism.

c) Let $Z^{\prime} \subset Z \subset X$ be a chain of closed subsets of $X$. Then for any coefficients $C$, there is a long exact sequence

$$
\begin{aligned}
\cdots \rightarrow H_{\mathrm{oc}}^{i}(X, Z, C) \rightarrow H_{\mathrm{oc}}^{i}\left(X, Z^{\prime}, C\right) \rightarrow & H_{\mathrm{oc}}^{i}\left(Z, Z^{\prime}, C\right) \\
& \rightarrow H_{\mathrm{oc}}^{i+1}(X, Z, C) \rightarrow \ldots
\end{aligned}
$$

d) Let $Z_{1}, Z_{2}, Z \subset X$ be closed, with $Z \supseteq Z_{1} \cap Z_{2}$. Then, for any coefficients $C$, the restriction map

$$
H_{\mathrm{oc}}^{*}\left(Z_{1} \cup Z_{2}, Z, C\right) \rightarrow H_{\mathrm{oc}}^{*}\left(Z_{1}, Z \cap Z_{1}, C\right) \oplus H_{\mathrm{oc}}^{*}\left(Z_{2}, Z \cap Z_{2}, C\right)
$$

is an isomorphism.

Proof. a) and b) are clear by closed Mayer-Vietoris; c) has nothing to do with the oc topology and is valid for any cohomology theory. The nicest way to prove d) is via a diagram chase in the derived category, which is left to the reader.

2.3. Lemma. Let $X$ be a scheme, $A$ a Zariski sheaf on $X$ and let $\pi$ denote the projection $X_{\mathrm{oc}} \rightarrow X_{\mathrm{Zar}}$ of the oc site of $X$ onto its Zariski 
site. Then, for $Y \in X_{\mathrm{oc}}$, the group of sections of $\pi^{*} A$ on $Y$ may be computed as

$$
H_{\mathrm{oc}}^{0}\left(Y, \pi^{*} A\right)=\lim _{\longrightarrow} \check{H}_{\mathrm{Zar}}^{0}\left(\left\{Z_{i}\right\}, \pi^{\sharp} A\right)
$$

where $\left\{Z_{i}\right\}$ runs through the finite closed covers of $Y$ and $\pi^{\sharp} A$ is the inverse image of $A$ as a presheaf. If $X$, hence $Y$, is Noetherian, we may replace the right hand side by $\check{H}_{\mathrm{Zar}}^{0}\left(\left\{Z_{i}\right\}, A\right)$, where $\left\{Z_{i}\right\}$ is the collection of irreducible components of $Y$.

Proof. This amounts to checking that the presheaf defined by the right hand side is indeed a sheaf in the oc topology. It suffices to check that it satisfies the sheaf condition for closed covers, which is true by construction, and for open covers, which is a simple computation left to the reader. The last claim simply follows from the fact that the irreducible components of $Y$ form a maximal closed cover of $Y$.

2.4. Lemma. Let $X$ be a locally irreducible scheme (all local rings of $X$ are irreducible). Then

a) Any open-closed cover of $X$ contains a Zariski open cover.

b) For any Zariski sheaf $A$ on $X$, the map $H_{\mathrm{Zar}}^{0}(X, A) \rightarrow H_{\mathrm{oc}}^{0}\left(X, \pi^{*} A\right)$ is an isomorphism.

c) The functor $\pi_{*}$ is exact.

d) The open-closed cohomology of $X$ coincides with its Zariski cohomology.

Proof. We may assume $X$ connected, hence irreducible. a) Any open subset $U$ of $X$ is also irreducible, hence any closed cover of $U$ is trivial. b) Follows from a) and lemma 2.3. c) We may assume $X$ local. Then $X$ has no nontrivial oc covers and the exactness of $\pi_{*}$ follows. d) follows from a), b) and c).

Examples of locally irreducible schemes are normal schemes, and in particular regular schemes.

2.5. Lemma. Let $X$ be a locally irreducible scheme and $Z_{1}, \ldots, Z_{n}$ a collection of closed subsets of $X$ such that the $Z_{i}$ and all their multiple intersections are locally irreducible. Then, for any complex of Zariski sheaves $C$ over $X$ and any $q \in \mathbb{Z}$, the natural map

$$
H_{\mathrm{Zar}}^{q}\left(X, Z_{1}, \ldots, Z_{n}, C\right) \rightarrow H_{\mathrm{oc}}^{q}\left(X, Z_{1}, \ldots, Z_{n}, C\right)
$$

is an isomorphism.

Proof. This immediately follows from lemma 2.4. 


\subsection{Normal crossing schemes.}

2.6. Definition. Let $T$ be a category of Noetherian locally irreducible $S$-schemes. A normal crossing $S$-scheme modelled on $T$ is a reduced Noetherian scheme $X$ with the following property: the intersection of any set of irreducible components of $X$ belongs to $T$ (or is empty).

This definition is related to that of $[10,2.8]$ and to Thomason's definition of a "bon assemblage" in [28, def. 3.1].

2.2.1. Two spectral sequences. Let $X$ be a normal crossing scheme modelled on $T$, and let $\left(X_{i}\right)_{i \in I}$ be the set of its irreducible components. For $J \subset I$, we write $X_{J}$ for $\bigcap_{i \in J} X_{i}$. To compute the oc cohomology of $X$, we enjoy two spectral sequences; given a complex of oc sheaves $C$ :

- The Mayer-Vietoris spectral sequence (of cohomological type).

$$
E_{1}^{p, q}=\bigoplus_{|J|=p+1} H_{\mathrm{oc}}^{q}\left(X_{J}, C\right) \Rightarrow H_{\mathrm{oc}}^{p+q}(X, C) .
$$

- The skeletal spectral sequence (of homological type). For $n \geq 0$, let

$$
S k^{n} X=\bigcup_{|J|=n+1} X_{J}
$$

This is the $n$-th coskeleton of $X$. Using Proposition $2.2 \mathrm{c}$ ), we get an exact couple, hence a spectral sequence

$$
E_{p, q}^{1}=H_{\mathrm{oc}}^{-p-q}\left(S k^{p} X, S k^{p+1} X, C\right) \Rightarrow H_{\mathrm{oc}}^{-p-q}(X, C) .
$$

Using Proposition $2.2 \mathrm{~d}$ ), we get the final form of the $E^{1}$-terms:

$$
E_{p, q}^{1}=\bigoplus_{|J|=p+1} H_{\mathrm{oc}}^{-p-q}\left(X_{J}, \bigcup_{K \supset J}^{|K|=p+2} X_{K}, C\right) .
$$

If $\tau$ is a site with underlying category $S, S^{\prime}$ another category and $u: S^{\prime} \rightarrow S$ a functor, we have the induced topology on $S^{\prime}$ [SGA4, III.3.1]. We shall only be interested in the case where $u$ is an inclusion, and will denote by $S_{\tau}^{\prime}$ the corresponding site with underlying category $S^{\prime}$. Let $T$ be as in Definition 2.6: the content of Lemma 2.4 is that the "change of topology" morphism

$$
\pi: T_{\mathrm{Oc}} \rightarrow T_{\mathrm{Zar}}
$$

is an isomorphism of sites. On the other hand: 
2.7. Proposition. Let $N c(T)$ be the category of normal crossing $S$ schemes modelled on $T$. Denote by $r: N c(T)_{\mathrm{Oc}} \rightarrow T_{\mathrm{Oc}}$ the corresponding morphism of sites. Then the restriction functor

$$
R r_{*}: D\left(A b\left(N c(T)_{\mathrm{Oc}}\right)\right) \rightarrow D\left(A b\left(T_{\mathrm{Oc}}\right)\right)
$$

has a right adjoint $R r^{!}$, given on the level of complexes by the formula

$$
C_{X}=\operatorname{Tot}\left(\bigoplus_{i \in I}\left(\iota_{i}\right)_{*} C_{i} \rightarrow \bigoplus_{J \subseteq I}^{|J|=2}\left(\iota_{J}\right)_{*} C_{J} \rightarrow \ldots\right)
$$

for $X \in N c(T)$, using the same notation as in 2.2.1 and writing $\iota_{J}$ for the inclusion $X_{J} \rightarrow X$ and $C_{J}$ for the restriction of $C$ to $X_{J}$.

Moreover, $R r_{*}$ and $R r^{!}$are inverse equivalences of categories.

Proof. Clearly, $(C, X) \mapsto C_{X}$ defines a triangulated functor $D\left(A b\left(T_{\mathrm{Oc}}\right)\right) \rightarrow D\left(A b\left(N c(T)_{\mathrm{Oc}}\right)\right)$, which is right inverse to $R r_{*}$. MayerVietoris for closed covers shows that it is also a left inverse. The fact that it is right adjoint to $R r_{*}$ will not be used here and is left to the reader.

2.8. Definition. For $C \in D\left(A b\left(T_{\text {Zar }}\right)\right)$, we shall denote $R r^{!} \pi^{*} C$ simply by $C_{\mathrm{oc}}$ and call it the canonical extension of $C$ to normal crossing schemes.

\subsection{Rationally constant complexes.}

From now on, $S=$ Spec $k$ for $k$ a field.

2.9. Definition. Let $\widetilde{S m} / k$ be the category of essentially smooth $k$ schemes. A normal crossing $k$-scheme modeled on $\widetilde{S m} / k$ will just be called a normal crossing $k$-scheme. The category of normal crossing $k$-schemes is denoted by $N c / k$.

If $C$ is a complex of sheaves of abelian groups on $(S m / k)_{\mathrm{Zar}}$, we shall still denote by $C$ its canonical extension to $(\widetilde{S m} / k)_{\text {Zar }}$, obtained by pull-back. As before, we shall write $C_{\mathrm{oc}}$, and sometimes $C$, for the (tautological) extension of the latter to $(\widetilde{S m} / k)_{\mathrm{Oc}}$ and the canonical extension of the latter to $(N c / k)_{\mathrm{Oc}}$ as in Proposition 2.7.

2.10. Definition. A complex of Zariski sheaves $C$ on $S m / k$ is rationally constant if it has the following two properties:

a) For any connected $X \in S m / k, C_{X} \rightarrow R j_{*} j^{*} C_{k(X)}$ is an isomorphism, where $j: \operatorname{Spec} k(X) \rightarrow X$ is the inclusion of the generic point.

b) For any extension $K / k$ of finite type and any rational extension $L / K, C_{K} \rightarrow R p_{*} C_{L}$ is an isomorphism, where $p: \operatorname{Spec} L \rightarrow \operatorname{Spec} K$ is 
the structural morphism.

The following lemma is clear.

2.11. Lemma. Let $C$ be a rationally constant complex of sheaves. a) Let $X \in N c / k$, and let $\left(Z_{i}\right)_{i \in I}$ be the set of its irreducible components. Assume that for any $J \subset I$, the intersection $Z_{J}$ of the corresponding components is a k-rational variety or is empty. Let $S .(X)$ be the simplicial complex corresponding to the $J \subset I$ such that $Z_{J} \neq \emptyset$. Then

$$
H_{\mathrm{oc}}^{*}(X, C) \simeq H_{\mathrm{oc}}^{*}\left(k, C \otimes C_{*}(S .(X))=H_{\mathrm{Zar}}^{*}\left(k, C \otimes C_{*}(S .(X))\right.\right.
$$

where $C_{*}(S .(X))$ is the chain complex associated to $S .(X)$.

b) Suppose $X$ is embedded as a closed subscheme in an essentially smooth rational $k$-scheme $Y$. Then

$$
H_{\mathrm{oc}}^{*}(Y ; X, C) \simeq H_{\mathrm{oc}}^{*}\left(k, C \otimes \widetilde{C}_{*}(S .(X))=H_{\mathrm{Zar}}^{*}\left(k, C \otimes \widetilde{C}_{*}(S .(X))\right.\right.
$$

where $\widetilde{C}_{*}(S .(X))$ is the reduced chain complex associated to $S .(X)$.

2.4. Local spheres and malleable complexes. We now introduce the objects $\square_{n}, \hat{\square}_{n}, \partial \hat{\square}_{n}, S_{n}, T_{n}$ of [9] and [10]. For more details on their combinatorics, the reader should consult $[10, \S 5]$. Recall their definitions:

- $\square_{n}$ denotes the affine space $\mathbf{A}_{k}^{n}$ (the $n$-cube).

- $\hat{\square}_{n}$ denotes the semi-localisation of $\square_{n}$ at the points $\left(\varepsilon_{1}, \ldots, \varepsilon_{n}\right)$, $\varepsilon_{j} \in\{0,1\}$ (the vertices of the $n$-cube): this is the semi-local $n$-cube.

- For $1 \leq i \leq n$ and $\varepsilon \in\{0,1\}, \square_{n}^{i, \varepsilon}$ denotes the $(n-1)$-face defined by the equation $t_{i}=\varepsilon ; \hat{\square}_{n}^{i, \varepsilon}=\square_{n}^{i, \varepsilon} \cap \hat{\square}_{n}$.

- $T_{n}=\left\{\hat{\square}_{n}^{i, \varepsilon} \mid 1 \leq i \leq n, \varepsilon \in\{0,1\}\right\}$.

- $\partial \hat{\square}_{n}=\bigcup_{T_{n}} \hat{\square}_{n}^{i, \varepsilon}$, the boundary of the semi-local $n$-cube.

- $T_{n}^{s}=$ the collection of the "first" $s$ members of $T_{n}$ for an appropriate order.

- $S_{n}=T_{n}^{2 n-1}$, the collection of all members of $T_{n}$, but one.

We also denote by $u T_{n}^{s}$ the union of all faces of $T_{n}^{s}$ (for example, $\left.u T_{n}^{2 n}=\partial \hat{\square}_{n}\right)$. When it is necessary to specify the ground field $k$, we put it as an index. One may think of $\partial \hat{\square}_{n}$ as a local $(n-1)$-sphere.

2.12. Proposition. Let $C$ be a complex of Zariski sheaves on $S m / k$. Let us just write $C$ for $C_{\mathrm{oc}}$. Then, for all $p \geq 0$ and $q \in \mathbb{Z}$,

a) If $H_{\mathrm{oc}}^{q}\left(\partial \hat{\square}_{p+1}, C\right)=0$, then $H_{\mathrm{oc}}^{q}\left(\hat{\square}_{p} ; \partial \hat{\square}_{p}, \bar{C}\right)=0$.

b) If $H_{\mathrm{oc}}^{q}\left(\hat{\square}_{m} ; \partial \hat{\square}_{m}, C\right)=0$ for all $m \leq p$, then $H_{\mathrm{oc}}^{q}\left(\partial \hat{\square}_{p+1}, C\right)=0$. 
c) Suppose that $C$ is bounded above, and let $d=\sup \left\{i \mid \mathcal{H}^{i}(C) \neq 0\right\}$. Moreover, suppose that $H^{q}\left(\hat{\square}_{p}, C\right)=0$ for $p \geq 0$ and $q>d^{1}$. Then, for any $s<2 p$ and any $q>d, H_{\mathrm{oc}}^{q}\left(u T_{p}^{s}, C\right)=0$.

d) For all $q \in \mathbb{Z}$ and all $p \geq 0$, there is a canonical map

$$
H_{\mathrm{oc}}^{q}\left(\partial \hat{\square}_{p}, C\right) \rightarrow H_{\mathrm{oc}}^{q+1}\left(\partial \hat{\square}_{p+1}, C\right) .
$$

Under the assumptions of $c$ ), this map is surjective for $q=d$ and bijective for $q>d$.

e) Under the assumptions of $c), H_{\mathrm{oc}}^{q}\left(\partial \hat{\square}_{p}, C\right)=0$ for $q>d$ and $p \leq$ $q-d$.

Proof. a) By proposition 2.22 b), the restriction map $H_{\mathrm{oc}}^{q}\left(\partial \hat{\square}_{p+1}, u S_{p+1}, C\right) \rightarrow H_{\mathrm{oc}}^{q}\left(\hat{\square}_{p}, \partial \hat{\square}_{p}, C\right)$ is an isomorphism. Since $S_{p+1}$ contains all faces but one, $H_{\mathrm{oc}}^{q}\left(\partial \hat{\square}_{p+1}, u S_{p+1}, C\right)$ is a functorial direct summand of $H_{\mathrm{oc}}^{q}\left(\partial \hat{\square}_{p+1}, C\right)$ (compare [10, Prop. 5.7]) and the claim follows.

b) follows from the skeletal spectral sequence of 2.2.1 applied to $\partial \hat{\square}_{p+1}$, by observing that a typical summand of a $E^{1}$-term is of the form $H_{\mathrm{oc}}^{-p-q}\left(\hat{\square}_{m} ; \partial \hat{\square}_{m}, C\right)$.

c) Applying [10, Prop. 5.7] with $F$ a Cartan-Eilenberg resolution of $C$, we get that the map $H_{\mathrm{oc}}^{q}\left(\hat{\square}_{p}, u T_{p}^{s}, C\right) \rightarrow H_{\mathrm{oc}}^{q}\left(\hat{\square}_{p}, C\right)$ is split injective for all $q \in \mathbb{Z}$, hence short exact sequences

$$
0 \rightarrow H_{\mathrm{oc}}^{q}\left(\hat{\square}_{p}, u T_{p}^{s}, C\right) \rightarrow H_{\mathrm{oc}}^{q}\left(\hat{\square}_{p}, C\right) \rightarrow H_{\mathrm{oc}}^{q}\left(u T_{p}^{s}, C\right) \rightarrow 0 .
$$

The map of d) comes from the Mayer-Vietoris exact sequence

$$
\begin{aligned}
H_{\mathrm{oc}}^{q}\left(u S_{p}, C\right) \oplus H_{\mathrm{oc}}^{q}\left(\hat{\square}_{p}, C\right) \rightarrow H_{\mathrm{oc}}^{q}\left(\partial \hat{\square}_{p}, C\right) \\
\rightarrow H_{\mathrm{oc}}^{q+1}\left(\partial \hat{\square}_{p+1}, C\right) \rightarrow H_{\mathrm{oc}}^{q+1}\left(u S_{p}, C\right) \oplus H_{\mathrm{oc}}^{q+1}\left(\hat{\square}_{p}, C\right)
\end{aligned}
$$

and its claimed surjectivity for $q=d$ and bijectivity for $q>d$ follow from c).

Finally, e) follows from d) by remarking that $\partial \hat{\square}_{1}$ is a disjoint union of 4 copies of Spec $k$.

2.13. Corollary. Let $C, d$ be as in Proposition 2.12 c). Then the following conditions are equivalent:

(i) There exists $N>d$ such that $H_{\mathrm{oc}}^{N}\left(\partial \hat{\square}_{p}, C\right)=0$ for all $p>0$.

(ii) Same as (i), with $N=d+1$.

(iii) For all $q>d$ and all $p>0, H_{\mathrm{oc}}^{q}\left(\partial \hat{\square}_{p}, C\right)=0$.

\footnotetext{
${ }^{1}$ This assumption is superfluous if $C$ verifies Gersten's conjecture: this will be the case for the cohomology theories we consider below, $c f$. Lemma $2.21 \mathrm{~b}$ ).
} 
Proof. (iii) $\Rightarrow$ (ii) $\Rightarrow$ (i) is clear. Let us show that (i) $\Rightarrow$ (iii). If $q \leq N$, the conclusion follows from Proposition 2.12 d). Suppose $q>N$. By Proposition $2.12 \mathrm{e}$ ), there is nothing to prove for $p \leq q-d$, and for $p>q-d$, we reapply Proposition $2.12 \mathrm{~d}$ ).

We now refine Proposition 2.12 d) and Corollary 2.13 as follows. Consider an arbitrary complex $C$ of Zariski sheaves on $S m / k$. Choose a Cartan-Eilenberg resolution $C_{\text {oc }} \rightarrow F$ of $C_{\text {oc }}$. For any $p>0$, the total complex associated to the sequence of chain complexes

$$
0 \rightarrow F\left(\partial \hat{\square}_{p+1}\right) \stackrel{\alpha_{p}}{\longrightarrow} F\left(\hat{\square}_{p}\right) \oplus F\left(u S_{p}\right) \rightarrow F\left(\partial \hat{\square}_{p}\right) \rightarrow 0
$$

is acyclic. In other words, the map

$$
\text { Cone }\left(\alpha_{p}\right) \rightarrow F\left(\partial \hat{\square}_{p}\right)
$$

is a quasi-isomorphism. Consider now the infinite diagram

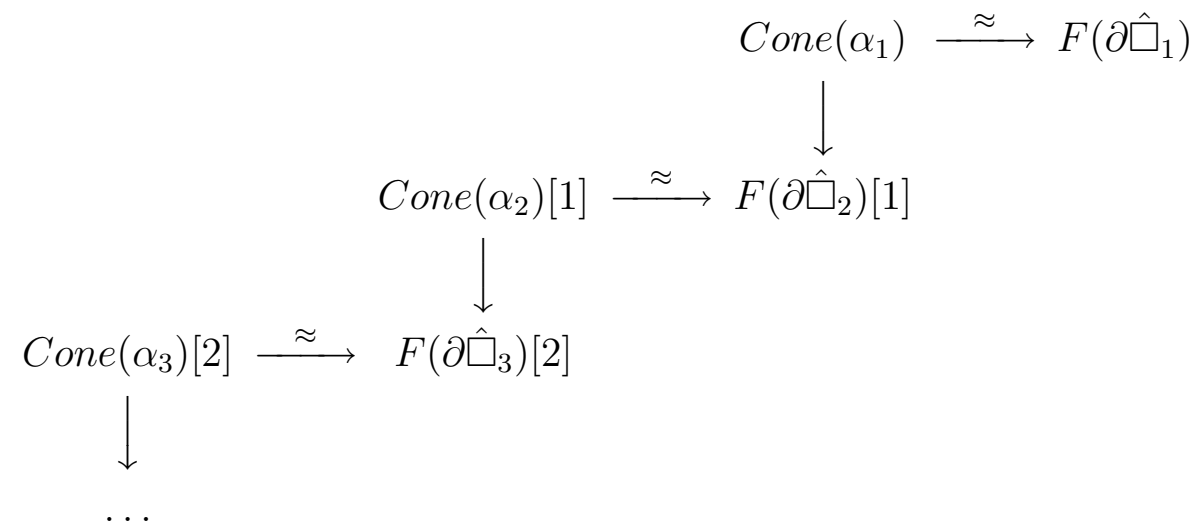

where the vertical maps are the obvious ones.

2.14. Definition. a) With notation as above, we denote by $C_{\infty}(k)$ the class in $D(A b)$ of the complex

$$
\text { Cone }\left(\bigoplus_{p} \text { Cone }\left(\alpha_{p}\right)[p-1] \rightarrow \bigoplus F\left(\partial \hat{\square}_{p+1}\right)[p]\right) .
$$

This is the type at infinity of $C$ evaluated on $k$.

b) We define a map in $D(A b)$

$$
R \Gamma(k, C) \rightarrow C_{\infty}(k)
$$

using the inclusion Spec $k \rightarrow \partial \hat{\square}_{1}$ given by the point $(0,0)$.

(The construction in a) is a mapping telescope, e.g. [4].)

2.15. Lemma. a) We have

$$
H^{i}\left(C_{\infty}(k)\right)=\lim _{\longrightarrow} H_{\mathrm{oc}}^{i+p}\left(\partial \hat{\square}_{p+1}, C\right)
$$


where the limit is taken according to the maps of Proposition 2.12 d).

b) The assignment $C \mapsto C_{\infty}(k)$ defines a triangulated functor from $D\left((S m / k)_{\text {Zar }}\right)$ to $D(A b)$, and $(2.1)$ is natural in $C$.

c) If $C$ is rationally constant (Definition 2.10), then (2.1) is an isomorphism.

d) Let $K / k$ be an extension, and define $C_{\infty}(K)$ in the same way as in Definition 2.14 after extension of scalars to $K$. Then the assignment $K \mapsto C_{\infty}(K)$ is natural in $K$, and so are the natural transformations (2.1). Moreover, $(C \stackrel{L}{\otimes} A)_{\infty}(k)=C_{\infty}(k) \stackrel{L}{\otimes} A$ for any constant complex of Zariski sheaves $A$.

e) For $C, d$ as in Proposition 2.12 c), the three conditions of Corollary 2.13 are also equivalent to the following:

(iv) $C_{\infty}(k)=0$.

Proof. The only nonobvious thing is c). Applying Lemma 2.11 a) to $X=\partial \hat{\square}_{p+1}$, we get for $p>0$

$$
H_{\mathrm{oc}}^{i+p}\left(\partial \hat{\square}_{p+1}, C\right) \simeq H^{i}(k, C) \oplus H^{i+p}(k, C)
$$

(notice that $S .(X)$ has the homotopy type of a $p$-sphere for $p>0$ ). The construction of the maps in Proposition 2.12 shows that the summands $H^{i+p}(k, C)$ go away in the limit.

2.16. Definition. Let $K / k$ be a finitely generated extension. A complex of Zariski sheaves is $K$-malleable if it verifies the assumptions of Proposition $2.12 \mathrm{c}$ ) and the equivalent conditions of Corollary 2.13 (also equivalent to condition (iv) in Lemma $2.15 \mathrm{e}$ )) with the ground field $k$ replaced by $K$. It is malleable if it is $K$-malleable for any $K$.

2.17. Remark. This is an unpleasant definition in that it is not intrinsic to $C$, which is defined as a complex of Zariski sheaves over smooth $k$ schemes. It would be very interesting to have a characterization of malleable complexes that only involves smooth schemes and not local spheres, or more generally to be able to compute $C_{\infty}$ out of a complex $C$ by using only smooth schemes. All that can be said from the outset is that malleable complexes form a thick (triangulated) subcategory of $D^{-}(A b(\widetilde{S m} / k))$, which is stable under derived tensor product by bounded above complexes of constant sheaves : this is obvious from Lemma $2.15 \mathrm{~d}$ ).

We shall later see nontrivial examples of malleable complexes.

2.18. Lemma. A malleable rationally constant complex of Zariski sheaves is quasi-isomorphic to 0. 
Proof. This is clear from the definition of malleability and Lemma $2.15 \mathrm{c})$.

\subsection{Homotopy invariant pure graded cohomology theories of complex-theoretic type.}

From now on, the field $k$ is perfect.

2.19. Definition. A homotopy invariant pure graded cohomology theory of complex-theoretic type over $k$ is a collection $(C(n))_{n \in \mathbb{Z}}$ of objects of $D^{-}\left(A b\left((S m / k)_{\text {Zar }}\right)\right)$ (the bounded above derived category of the category of abelian sheaves on the big smooth Zariski site of Spec $k$ ), satisfying the following axioms:

(i) Homotopy invariance. For any $n, C(n) \rightarrow R f_{*} f^{*} C(n)$ is an isomorphism, where $f$ is the structural map $\mathbf{A}_{k}^{1} \rightarrow$ Spec $k$.

(ii) Purity. Let $i: Z \rightarrow X$ be a smooth pair of pure codimension $c$. Then there exists for all $n$ an isomorphism

$$
\alpha(i): C(n-c)_{Z}[-2 c] \stackrel{\sim}{\longrightarrow} R i^{!} C(n)_{X}
$$

which is contravariant for étale morphisms of smooth pairs of pure codimension $c$.

The integer $n$ in $C(n)$ is called the weight.

$C$ is said to admit étale transfers if, for any finite étale morphism $f: Y \rightarrow X$ of degree $d$, one is given a map

$$
\operatorname{Tr}_{f}: f_{*} C(n)_{Y} \rightarrow C(n)_{X}
$$

such that the composition

$$
C(n)_{X} \longrightarrow f_{*} C(n)_{Y} \stackrel{T r_{f}}{\longrightarrow} C(n)_{X}
$$

is multiplication by $d$.

In the sequel, we abbreviate homotopy invariant pure graded cohomology theory of complex-theoretic type into cohomology theory.

Cohomology theories over $k$ form a category for morphisms respecting the purity structures. This category is not quite a triangulated category; however, if $A(n) \rightarrow B(n)$ is a morphism of cohomology theories and $(C(n))_{n \in \mathbb{Z}}$ is a collection of corresponding cones, then $C(*)$ can be provided with a structure of a cohomology theory. Indeed, let $i: Z \rightarrow X$ be a smooth pair of codimension $c$. Consider the restriction of $C(n)$ to the small étale site $X_{\text {ét }}$ of $X$. By one axiom of triangulated categories, there exists a morphism on $X_{\text {ét }}$

$$
\beta(i): i_{*} C(n-c)_{Z}[-2 c] \rightarrow C(n)_{X}
$$


commuting with the corresponding morphisms for $A$ and $B$, and the adjoint morphism $\alpha(i): C(n-c)_{Z}[-2 c] \rightarrow R i^{!} C(n)_{X}$ is an isomorphism.

2.20. Examples.

(1) For all $n \geq 0$, let $\mathbb{Z}(n)$ be the object of $D^{-}\left(A b\left((S m / k)_{\text {Zar }}\right)\right)$ defined in theorem 1.17. By theorem 1.2, $(\mathbb{Z}(n))_{n \in \mathbb{Z}}$ defines a cohomology theory, called motivic cohomology.

(2) If $C(*)$ is a cohomology theory and $A$ is a complex of abelian groups, then $C(*) \stackrel{L}{\otimes} A$ is another cohomology theory.

(3) Truncated étale cohomology with finite coefficients defines a cohomology theory. First, let $m$ be prime to char $k$ and let $B / m(n)=\tau_{\leq n} R \alpha_{*} \mu_{m}^{\otimes n}$. Then homotopy invariance and purity follow from Gersten's conjecture applied to the cohomology sheaves of $B / m(n)$ and the same properties for étale cohomology [6, item (5) of 7.3]. Using Lemma 1.21 and Gersten's conjecture again applied to motivic cohomology, one sees that (1.7) defines a morphism of cohomology theories.

(4) The situation is similar for truncated Hodge-Witt cohomology. If char $k=p>0$ and $r>0$, let $B / p^{r}(n)=\tau_{\leq n} R \alpha_{*}\left(\nu_{r}(n)[-n]\right)=$ $\alpha_{*} \nu_{r}(n)[-n]$, where $\nu_{r}(n)$ is the étale logarithmic Hodge-Witt sheaf of length $r$ and weight $n$. Then purity follows from [11, th. 3.5.8] and homotopy invariance may be deduced from [12, cor. 1.6] and [6, item (5) of 7.3]. In this case, the definition of the cycle class map from motivic cohomology to truncated Hodge-Witt cohomology is particularly simple and is given once again via Gersten's conjecture, see [9, proof of th. 8.3].

(5) $l$-adic cohomology defines a cohomology theory, and the $l$-adic cycle map (1.8) is a morphism of cohomology theories (use Lemma 1.21 again). This morphism will be studied in the next section in the case where $k$ is a finite field.

Here are a few results on cohomology theories.

2.21. Lemma. Let $C(*)$ be a cohomology theory. Then:

a) $C(*)$ verifies Nisnevich excision for closed immersions in ambient smooth $k$-schemes.

b) $C(*)$ verifies Gersten's conjecture: for any finitely generated extension $K / k$, any semi-local smooth $K$-scheme $X$ and any $(i, n) \in \mathbb{Z} \times \mathbb{Z}$, 
we have a long exact sequence of abelian groups:

$$
\begin{aligned}
0 \rightarrow H^{i}(X, C(n)) \rightarrow \bigoplus_{x \in X^{(0)}} H^{i} & (K(x), C(n)) \\
& \rightarrow \bigoplus_{x \in X^{(1)}} H^{i-1}(K(x), C(n-1)) \rightarrow \ldots
\end{aligned}
$$

Proof. a) This is obvious in the case of a smooth pair of pure codimension, then for an arbitrary smooth pair, and then in general by Noetherian induction (use the singular locus of the closed subset).

b) We may reduce to $K=k$ by Quillen's trick: $X$ is a semilocalisation of some smooth $k$-scheme. It now follows from $[6$, Cor. 5.1.11] that the Cousin complexes give resolutions of the groups $H^{i}(X, C(n))$ (axioms $\mathrm{COH} 1$ and $\mathrm{COH} 3$ of loc. cit. are satisfied). Using the perfectness of $k$, we may finally use property (ii) in Definition 2.19 to replace the terms of the Cousin resolution by terms as they appear in Lemma 2.21.

2.22. Proposition. Let $C(*)$ be a cohomology theory over $k$. Assume that $C(m)=0$ for $m<n$. Then $C(n)$ is rationally constant.

Proof. In the first case, property a) of Definition 2.10 follows immediately from Lemma 2.21, and property b) follows from a) and homotopy invariance.

Finally, the following proposition shows how to "toggle" between function fields and smooth projective varieties.

2.23. Proposition. Assume $k$ perfect, and let $f: A \rightarrow B$ be a morphism of cohomology theories over $k$. Assume that $A(n)=B(n)=0$ for $n$ small enough. Consider the following conditions:

(i) $f$ is an isomorphism.

(ii) For any finitely generated extension $K / k$, any $n \in \mathbb{Z}$ and any $i \in \mathbb{Z}, f_{*}: H^{i}(K, A(n)) \rightarrow H^{i}(K, B(n))$ is an isomorphism.

(iii) For any smooth projective $k$-variety $X$, any $n \in \mathbb{Z}$ and any $i \in \mathbb{Z}, f_{*}: H^{i}(X, A(n)) \rightarrow H^{i}(X, B(n))$ is an isomorphism.

Then (i) $\Longleftrightarrow$ (ii) $\Rightarrow$ (iii). If char $k=0$, then (iii) $\Rightarrow$ (i). This conclusion also holds in characteristic $p>0$ if

a) the cohomology sheaves of the cone of $f$ are sheaves of $\mathbb{Q}$-vector spaces, and

b) $A$ and $B$ have étale transfers and $f$ commutes with these transfers. 
Proof. For (i) $\Longleftrightarrow$ (ii), we immediately reduce to $A=0$ by considering the cone of $f$. Then the equivalence follows from Proposition 2.22 a) by induction on the weight.

The implication (i) $\Rightarrow$ (iii) is trivial. The converse is an application of resolution of singularities in characteristic 0 , and of de Jong's alteration theorem [14] in characteristic $p$ : let us just give the latter (the characteristic 0 case is similar and simpler).

We argue by induction on the weight $n$, hence may assume that $A(m) \stackrel{\sim}{\longrightarrow} B(m)$ for $m<n$. Let $C(n)$ be the cone of $f_{n}$. Since $k$ is perfect, a Whitney stratification argument shows that, for $U$ an open subset of a smooth $k$-scheme $X$, the map $H^{i}(X, C(n)) \rightarrow H^{i}(U, C(n))$ is an isomorphism for all $i$ (if $X-U$ is smooth, this follows by purity). Moreover, if $\widetilde{U} \rightarrow U$ is an étale covering, conditions a) and b) show that the maps $H^{i}(U, A(n)) \rightarrow H^{i}(\widetilde{U}, A(n))$ and $H^{i}(U, B(n)) \rightarrow H^{i}(\widetilde{U}, B(n))$ are compatible split injections. It follows that, if $H^{i}(\widetilde{U}, A(n)) \stackrel{\sim}{\longrightarrow}$ $H^{i}(\widetilde{U}, B(n))$, then $H^{i}(U, A(n)) \stackrel{\sim}{\longrightarrow} H^{i}(U, B(n))$. The conclusion now follows from [14].

(The reason why we cannot directly argue with $C(n)$ in the proof of (iii) $\Rightarrow$ (i) is that, while it is clear that $C(n)$ can be provided with "transfers", it is not clear that we may choose these "transfers" to verify the last condition in Definition 2.19.)

2.24. Theorem. Let $C(n)$ be a cohomology theory over $k$. Assume that $C(m)=0$ for $m<n$. Assume that $C(n)$ is bounded above. Then $C(n)=0$ if and only if $C(n)$ is malleable.

Proof. Necessity is obvious. For sufficiency, note that $C(n)$ is rationally constant by Proposition 2.22. The conclusion now follows from Lemma 2.18.

2.25. Remark. By Lemma $2.21 \mathrm{~b}), C(n)$ satisfies the unpleasant assumption of Proposition $2.12 \mathrm{c}$ ) whether or not it is malleable.

2.26. Corollary. Let $f_{n}: A(n) \rightarrow B(n)$ be a morphism of cohomology theories. Assume that $f_{m}$ is an isomorphism for $m<n$. Suppose that $A(n)$ and $B(n)$ are both bounded above. Then $f_{n}$ is an isomorphism if and only if its cone is malleable.

2.6. The case of motivic cohomology. In [9, cor. 4.4], Geisser and Levine prove the following fundamental theorem:

2.27. Theorem. Let $n>0$. For any $q>n$ and any $p \geq 0$, one has

$$
H_{\mathrm{Zar}}^{q}\left(\hat{\square}_{p}, T_{p}, \mathbb{Z}(n)\right)=0 \text {. }
$$


Applying lemmas 2.5 and $2.12 \mathrm{~b}$ ), we get the following variant:

2.28. Theorem. For any $n>0, \mathbb{Z}(n)$ is malleable.

Note that theorems 2.27 and 2.28 are wrong for $n=0$, since $\mathbb{Z}(0)=$ $\mathbb{Z}[0]$ is rationally constant and nonzero ( $c f$. Lemma 2.18).

2.29. Corollary. For any bounded above complex A of abelian groups, $\mathbb{Z}(n) \stackrel{L}{\otimes} A$ is malleable (compare Remark 2.17).

From corollaries 2.26 and 2.29, we deduce:

2.30. Proposition. Let $(B(n))_{n \in \mathbb{Z}}$ be a cohomology theory such that all $B(n)$ are bounded above and $B(n)=0$ for $n<0$, and let $A$ a bounded above complex of abelian groups, acyclic in degree $>d$, and let $f_{n}$ : $\mathbb{Z}(n) \stackrel{L}{\otimes} A \rightarrow B(n)$ be a morphism of cohomology theories. Suppose that $f_{0}$ is an isomorphism. Then the following conditions are equivalent.

(i) For all $n>0, f_{n}$ is an isomorphism.

(ii) For all $n>0, B(n)$ is malleable.

(iii) For any finitely generated extension $K / k$, any $n>0$ and any $p>0$, the map

$$
H_{\mathrm{oc}}^{n+d}\left(\left(\partial \hat{\square}_{p}\right)_{K}, \mathbb{Z}(n) \stackrel{L}{\otimes} A\right) \stackrel{f_{n}}{\longrightarrow} H_{\mathrm{oc}}^{n+d}\left(\left(\partial \hat{\square}_{p}\right)_{K}, B(n)\right)
$$

is surjective.

Proof. Let $C(n)$ be the cone of $f_{n}$. The malleability of $\mathbb{Z}(n) \stackrel{L}{\otimes} A$ shows that conditions (ii) and (iii) are both equivalent to $C(n)$ being malleable. This in turn is equivalent to (i) by Corollary 2.26.

\subsection{Smoothable complexes.}

2.31. Definition. Let $C$ be bounded above complex of sheaves on $S m / k$, and let $d=\sup \left\{i \mid \mathcal{H}^{i}(C) \neq 0\right\}$. We say that $C$ is smoothable if the following holds:

For any finitely generated extension $K / k$, any $p>0$ and any $x \in$ $H_{\text {oc }}^{d}\left(\left(\partial \hat{\square}_{p}\right)_{K}, C\right)$, there exists an essentially smooth semi-local $K$-scheme $U$ and a map $g:\left(\partial \hat{\square}_{p}\right)_{K} \rightarrow U$ such that $x \in \operatorname{Im} g^{*}$.

2.32. Lemma. a) If $C$ is malleable, it is smoothable.

b) $\mathbb{Z}(n) \stackrel{L}{\otimes} A$ is smoothable for any $n>0$ and any bounded above complex of abelian groups $A$. 
Proof. a) The definition of malleability and Proposition 2.12 a) imply that the restriction map $H_{\mathrm{oc}}^{d}\left(\left(\hat{\square}_{p}\right)_{K}, C\right) \rightarrow H_{\mathrm{oc}}^{d}\left(\left(\partial \hat{\square}_{p}\right)_{K}, C\right)$ is surjective.

b) This follows from a) and Corollary 2.29.

2.33. Remark. It is obvious that $\mathbb{Z}(0)=\mathbb{Z}$ is also smoothable. In view of Lemma 2.18, this shows that malleability is strictly stronger than smoothability. On the other hand, $C=\mathbb{Z}[0] \oplus \mathbb{Z}[-1]$ is clearly not smoothable: indeed, $H^{1}\left(\partial \hat{\square}_{2}, C\right)=H^{1}(k, C) \oplus \mathbb{Z}$ while $H^{1}(U, C)=$ $H^{1}(k, C)$ for any smooth $U$. This shows that the notion of a smoothable complex is much more ad hoc than that of a malleable complex.

2.34. Theorem. Let $(B(n))_{n \in \mathbb{Z}}$ be a cohomology theory such that all $B(n)$ are bounded above and $B(n)=0$ for $n<0$, let $A$ a bounded above complex of abelian groups acyclic in degree $>d$ and let $f_{n}$ : $\mathbb{Z}(n) \stackrel{L}{\otimes} A \rightarrow B(n)$ be a morphism of cohomology theories. Assume that $f_{0}$ is an isomorphism. Then the following conditions are equivalent:

(i) For all $n>0, f_{n}$ is an isomorphism.

(ii) For all $n>0, B(n)$ is malleable.

(iii) For all $n>0, B(n)$ is smoothable and the map

$$
H^{n+d}(K, \mathbb{Z}(n) \stackrel{L}{\otimes} A) \rightarrow H^{n+d}(K, B(n))
$$

is surjective for any finitely generated extension $K / k$.

Proof. The equivalence between (i) and (ii) has been seen in Proposition 2.30. In view of Lemma 2.32, it is clear that (i) $\Rightarrow$ (iii). For the converse, let us show that condition (iii) of Theorem 2.34 implies condition (iii) of proposition 2.30.

Let $x \in H_{\mathrm{oc}}^{n+d}\left(\left(\partial \hat{\square}_{p}\right)_{K}, B(n)\right)$. By assumption, there exists an essentially smooth semi-local $K$-scheme $U$ and a map $g:\left(\partial \hat{\square}_{p}\right)_{K} \rightarrow U$ such that $x \in \operatorname{Im} g^{*}$.

Let $E$ be the function field of $U$ and $C(n)$ the cone of $f_{n}$. By (iii), we have $H^{n+d}(E, C(n))=0$ (use the fact that $H^{n+d+1}(E, \mathbb{Z}(n) \stackrel{L}{\otimes} A)=0$ ). Now Lemma $2.21 \mathrm{~b}$ ) implies that $H^{n+d}(U, C(n))=0$, hence the map $H^{n+d}(U, \mathbb{Z}(n) \stackrel{L}{\otimes} C) \rightarrow H^{n+d}(U, B(n))$ is surjective. Therefore $x$ comes from an element of $H_{\mathrm{oc}}^{n+d}\left(\left(\partial \hat{\square}_{p}\right)_{K}, \mathbb{Z}(n) \stackrel{L}{\otimes} A\right)$, as desired.

2.35. Scholium of small enlightenment. In [28], Thomason proves a theorem having a formal analogy with Theorem 2.34, for algebraic $K$ theory instead of motivic cohomology. Let us point out the similarities and differences: 
(1) In both cases, the proof relies on a suspension argument using normal crossing schemes (called bons assemblages in Thomason). This includes extending the theories at hand, defined on smooth varieties, to normal crossing schemes. In this respect, [28, Prop. 3.5] is analogous to our Definition 2.8 (resting on Proposition 2.7).

(2) The reduction to $K_{0}$ in $[28,4.4]$ is related to condition (iii) in Proposition 2.30 or Theorem 2.34.

(3) Here we have to resort to "local spheres", while Thomason merely uses "global spheres".

(4) Here, the key property of a cohomology theory is the mysterious malleability condition; in [28] there is no analogue of this property. Instead, the cohomology theory to be compared to $K$-theory is supposed to receive units in a way compatible to the way in which $K$-theory does: this allows Thomason to prove his Lemme critique 4.1. Maybe the counterpart here is the appearance of units in the proofs of malleability in Corollary 2.38 below?.

(5) To prove his Lemma 4.1, Thomason uses the fact that algebraic $K$-theory is a priori defined on normal crossing schemes $X$, and uses the comparison map $K_{0}(X) \rightarrow K H_{0}(X)$ where $K H$ is the canonical extension of $K$-theory to normal crossing schemes. Here, I have no idea what should be motivic cohomology of normal crossing schemes (not extended), supposing it exists.

(6) The main difference between [28] and this paper is, of course, that the comparison maps go in opposite directions: from motivic cohomology to another cohomology theory in our case, from another cohomology theory to algebraic $K$-theory in Thomason's case. So the two results are completely different. A more minor difference is that, here, we conclude to an isomorphism while Thomason merely concludes to a surjectivity result.

(7) Finally, at the moment one does not know any nontrivial example of a cohomology theory verifying Thomason's hypotheses.

\subsection{Motivic cohomology with finite coefficients.}

2.36. Lemma. Let $m$ be an integer prime to char $k$, and let $n \geq 0$.

a) For any normal crossing scheme $X$, there exist canonical maps

$$
H_{\mathrm{oc}}^{q}\left(X, B / m(n)_{\mathrm{oc}}\right) \rightarrow H_{\text {ét }}^{q}\left(X, \mu_{m}^{\otimes n}\right) .
$$

Here $B / m(n)=\tau_{\leq n} R \alpha_{*} \mu_{m}^{\otimes n}$ is truncated étale cohomology ( $\alpha$ is the projection from the big smooth étale site to the big smooth Zariski site), and $B / m(n)_{\mathrm{oc}}$ is its canonical extension to normal crossing schemes 
given by proposition 2.7.

b) These maps are isomorphisms for $q \leq n$.

c) For all $n \geq 0, B / m(n)$ is smoothable.

Proof. a) Since the map clearly exists for $X$ smooth (see lemma 2.4), the claim amounts to the fact that étale cohomology with finite coefficients satisfies Mayer-Vietoris for closed covers. This follows easily from the proper base change theorem (for a closed immersion!).

b) When $X$ is essentially smooth, the statement follows from a comparison of the hypercohomology spectral sequence yielding the left hand side with the Leray spectral sequence for $\alpha$ yielding the right hand side. In general, it follows from the smooth case by a comparison of two Mayer-Vietoris spectral sequences (for the closed cover given by the irreducible components of $X$ ).

c) This follows from b) and Gabber's rigidity theorem [7].

2.37. Lemma. Let $p=$ char $k, n \geq 0, s \geq 1$ and let

$$
B / p^{s}(n)=\tau_{\leq n}\left(R \alpha_{*} \nu_{s}(n)[-n]\right)=\left(\alpha_{*} \nu_{s}(n)\right)[-n]
$$

be truncated logarithmic de Rham-Witt cohomology. Then $B / p^{s}(n)$ is smoothable.

Proof. The smoothability condition holds for the global sections of $\nu_{s}(n)$ for the Zariski topology by [9, prop. 7.3]. Now, by [9, cor. 6.2], the map

$$
H_{\mathrm{Zar}}^{0}\left(\partial \hat{\square}_{m+1}, S_{m+1}, \nu^{n}\right) \rightarrow H_{\mathrm{Zar}}^{0}\left(\hat{\square}_{m}, T_{m}, \nu^{n}\right)
$$

is surjective for any $m \geq 0$. By proposition 2.2, lemma 2.5 and Proposition $2.22 \mathrm{~b}$ ), this translates into the surjectivity of the map

$$
H_{\mathrm{Zar}}^{0}\left(\partial \hat{\square}_{m+1}, S_{m+1}, \nu^{n}\right) \rightarrow H_{\mathrm{oc}}^{0}\left(\partial \hat{\square}_{m+1}, S_{m+1}, \nu^{n}\right) .
$$

But [10, prop. 5.7 1.] applied with $F=\nu^{n}$ implies that the sequence

$$
\nu^{n}\left(\partial \hat{\square}_{m+1}\right) \rightarrow \bigoplus_{(i, \varepsilon) \neq(n, 1)} \nu^{n}\left(\hat{\square}_{m+1}^{i, \varepsilon}\right) \rightarrow \bigoplus_{(i, \varepsilon) \neq\left(i^{\prime}, \varepsilon^{\prime}\right)} \nu^{n}\left(\hat{\square}_{m+1}^{i, \varepsilon} \cap \hat{\square}_{m+1}^{i^{\prime}, \varepsilon^{\prime}}\right)
$$

is exact. From this, one easily deduces that the map

$$
H_{\text {Zar }}^{0}\left(\partial \hat{\square}_{m+1}, \nu_{s}(n)\right) \rightarrow H_{\mathrm{oc}}^{0}\left(\partial \hat{\square}_{m+1}, \nu_{s}(n)\right)
$$

is surjective.

2.38. Corollary. Let $k$ be a field.

a) [26], [10] Let $l$ be a prime number $\neq$ char $k$. Then the following conditions are equivalent: 
(i) (Beilinson-Lichtenbaum conjecture) The map (1.7)

$$
\mathbb{Z} / l^{s}(n) \rightarrow B / l^{s}(n)
$$

is an isomorphism for all $n \geq 0$ and all $s \geq 1$.

(ii) For all $s \geq 1$ and $n>0, B / l^{s}(n)$ is malleable.

(iii) (Weak Bloch-Kato conjecture) For any finitely generated extension $K / k$ and any $n>0$, the Galois symbol $K_{n}^{M}(K) / l \rightarrow$ $H^{n}\left(K, \mu_{l}^{\otimes n}\right)$ is surjective.

b) [9] For $p=$ char $k$ and $s \geq 1$, the map

$$
\mathbb{Z} / p^{s}(n) \rightarrow B / p^{s}(n)
$$

is an isomorphism for all $n \geq 0$. The complex $B / p^{s}(n)$ is malleable for all $s \geq 1$ and all $n>0$.

Proof. Both theories a) and b) are cohomology theories and the maps are maps of cohomology theories (example 2.20). Since everything commutes with direct limits, we may assume that $k$ is the prime field, and in particular is perfect. The maps of a) and b) are clearly isomorphisms for $n=0$.

In view of Lemma $2.36 \mathrm{c}$ ), a) follows from Theorem 2.34 for $s=1$, hence for all $s$ by a well-known inductive argument due to Tate.

In b), it is enough to check that condition (iii) of Theorem 2.34 is verified. Smoothability is Lemma 2.37 and the surjectivity condition is a theorem of Kato [19, prop. 1]. The fact that $B / p^{s}(n)$ is malleable now follows from Theorem 2.34 .

\section{Applichtion to algebraic CyCles over $\mathbb{F}_{p}$}

We fix two prime numbers $p, l$, with $l \neq p$. In this section, $k=\mathbb{F}_{p}$.

3.1. The modified $l$-adic motivic cycle class map. We want to study how close the map (1.8)

$$
\alpha^{*} \mathbb{Z}(n) \stackrel{L}{\otimes} \mathbb{Z}_{l} \rightarrow \mathbb{Z}_{l}(n)^{c}(n \geq 0)
$$

is to being an isomorphism. At the outset, we see that it is not one for $n=0$ (see Proposition 3.7 below). The cheapest way to correct this problem is to modify (1.8) as follows: we take the composition

$$
\alpha^{*} \mathbb{Z}(n) \stackrel{L}{\otimes} \mathbb{Z}_{l}(0)^{c} \rightarrow \mathbb{Z}_{l}(n)^{c} \stackrel{L}{\otimes} \mathbb{Z}_{l}(0)^{c} \rightarrow \mathbb{Z}_{l}(n)^{c}
$$

where the last map is given by cup-product in l-adic cohomology. We call this composition $\Phi_{n}$ : this is the modified l-adic motivic cycle class map. At the very least, $\Phi_{0}$ is now an isomorphism. 
3.2. The conjectures. They are the following:

3.1. Conjecture. For any smooth, projective variety $X$ over $\mathbb{F}_{p}$ and any $n \geq 0$,

a) (Tate conjecture) The order of the pole of the zeta function $\zeta(X, s)$ at $s=n$ is equal to the rank of $A_{\text {num }}^{n}(X)$, the group of cycles of codimension $n$ on $X$ modulo numerical equivalence.

b) (Beilinson conjecture) Rational and numerical equivalences agree on cycles of codimension $n$ on $X$ (after tensoring with $\mathbb{Q}$ ).

3.2. Conjecture. For all $n \geq 0$, the modified l-adic motivic cycle class map

$$
\Phi_{n}: \alpha^{*} \mathbb{Z}(n) \stackrel{L}{\otimes} \mathbb{Z}_{l}(0)^{c} \rightarrow \mathbb{Z}_{l}(n)^{c}
$$

is an isomorphism in $D\left(\left(S m / \mathbb{F}_{p}\right)\right.$ ét $)$.

3.3. Conjecture. For all $n>0$, the complex $\mathbb{Q}_{l}(n)^{c}$ is malleable (cf. Definition 2.16).

Our main result is:

3.4. Theorem. Conjecture 3.1 $\Longleftrightarrow$ Conjecture 3.2 2 Conjecture 3.3.

We remark:

3.5. Corollary. Conjectures 3.2 and 3.3 are independent of $l$.

Indeed, $l$ does not appear in the formulation of Conjecture 3.1.

The proof of Theorem 3.4 will be given in the next two subsections. Before, we prove two useful results.

3.6. Lemma. Conjecture 3.2 is true if and only if it is true after tensoring with $\mathbb{Q}$.

Indeed, Theorem 1.22 shows that conjecture 3.2 is true after tensoring with $\mathbb{Z} / l$ (in the derived sense). The claim now follows from a classical five lemma argument.

3.7. Proposition. On $S m / \mathbb{F}_{p}, \mathbb{Q}_{l}(n)^{c}$ is acyclic in degrees $\notin[n, 2 n+1]$. In particular, $\mathbb{Q}_{l}(n)^{c}=0$ for $n<0$. Moreover, $\mathbb{Q}_{l}(0)^{c} \simeq \mathbb{Q}_{l}[0] \oplus \mathbb{Q}_{l}[-1]$, and cup-product by e: $\mathcal{H}^{0}\left(\mathbb{Q}_{l}(0)^{c}\right) \rightarrow \mathcal{H}^{1}\left(\mathbb{Q}_{l}(0)^{c}\right)$ is an isomorphism, where $e$ is the generator of $H_{\text {cont }}^{1}\left(\mathbb{F}_{p}, \mathbb{Z}_{l}\right) \simeq \operatorname{Hom}_{\text {cont }}\left(\operatorname{Gal}\left(\overline{\mathbb{F}}_{p} / \mathbb{F}_{p}\right), \mathbb{Z}_{l}\right)$ which maps the arithmetical Frobenius to 1.

Proof. This follows from [16, Th. 1 and Remark 6]. 
In view of the last statement of Proposition 3.7, for any smooth $\mathbb{F}_{p}$-variety $X, \Phi_{n}$ defines homomorphisms

$$
H^{q}(X, \mathbb{Q}(n)) \otimes \mathbb{Q}_{l} \oplus H^{q-1}(X, \mathbb{Q}(n)) \otimes \mathbb{Q}_{l} \rightarrow H_{\text {cont }}^{q}\left(X, \mathbb{Q}_{l}(n)\right)
$$

which are such that the diagram

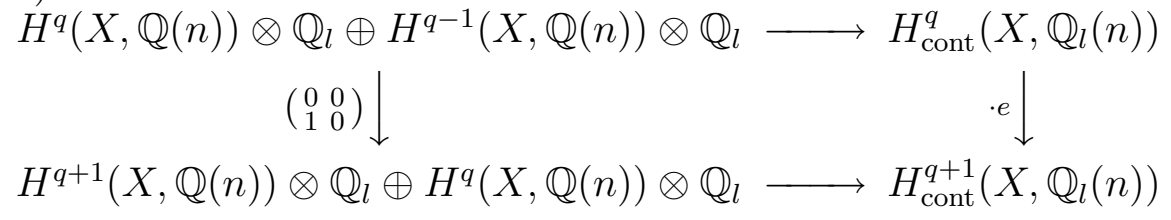

commutes for all $q \in \mathbb{Z}$.

3.3. Equivalence between Conjecture 3.2 and Conjecture 3.3. By Lemma 3.6, it suffices to check that the assumptions of Theorem 2.34 hold for $A=R \alpha_{*} \mathbb{Q}_{l}(0)^{c}$ and $B(n)=R \alpha_{*} \mathbb{Q}_{l}(n)^{c}$. We have already seen that the $R \alpha_{*} \Phi_{n}$, and hence the $R \alpha_{*} \Phi_{n} \otimes \mathbb{Q}$, define a morphism of cohomology theories. Then, Proposition 3.7 shows that $B(n)$ is bounded above for all $n$ and is 0 for $n<0$. Finally, $\Phi_{0}$ is tautologically an isomorphism.

\subsection{Equivalence between Conjecture 3.1 and Conjecture 3.2.}

Let $X \in S m / \mathbb{F}_{p}$ and $n \geq 0$. To evaluate Conjecture 3.2 on $X$ in weight $n$ is to consider whether the induced morphism $H_{\text {cont }}^{q}\left(X, \Phi_{n}\right)$ is an isomorphism for any $q \in \mathbb{Z}$. By Lemma 3.6, Conjecture 3.2 holds if and only if it holds when evaluated on $X$, for any $X \in S m / \mathbb{F}_{p}$, after tensoring with $\mathbb{Q}$.

3.8. Lemma. Conjecture 3.2 holds if and only if it holds when evaluated on $X$ in weight $n$, for any projective $X \in S m / \mathbb{F}_{p}$ and any $n \geq 0$, after tensoring with $\mathbb{Q}$.

Proof. This follows from Proposition 2.23 and the remarks preceding the Lemma.

3.9. Proposition. Let $X / \mathbb{F}_{p}$ be a smooth, projective variety. Then Conjecture 3.2 holds when evaluated on $X$ in weight $n$ (after tensoring with $\mathbb{Q})$ if and only if

(i) $H^{i}(X, \mathbb{Q}(n))=0$ for $i \neq 2 n$.

(ii) The cohomological Tate conjecture holds for $X$ in codimension $n$ and rational equivalence equals homological equivalence in codimension $n$ over $X$ (for $\mathbb{Q}_{l}$ coeffients).

(iii) Condition $S^{n}(X)$ of $[27]$ holds. 
Proof. Recall that $H^{q}(X, \mathbb{Z}(n))=0$ for $q>2 n$ and that $H^{2 n}(X, \mathbb{Z}(n))$ $\simeq C H^{n}(X)$ (Proposition 1.6 (i) and (ii)), and also that the map $H^{q}(X, \mathbb{Q}(n)) \rightarrow H_{\text {ét }}^{q}(X, \mathbb{Q}(n))$ is an isomorphism for all $q$ (Proposition 1.18). We first show that Conjecture 3.2 implies conditions (i)-(iii). Since $X$ is smooth projective, the right hand side of (3.1) is 0 for $q \neq 2 n, 2 n+1[5$, th. 2]: this implies (i). For $q=2 n, 2 n+1$, we then get as a special case of (3.2) a commutative diagram

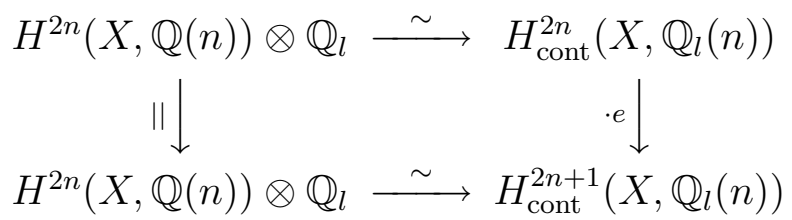

and by lemma 1.23, the top horizontal map is the $l$-adic cycle map. On the other hand, by [22, prop. 6.5], we have a commutative diagram

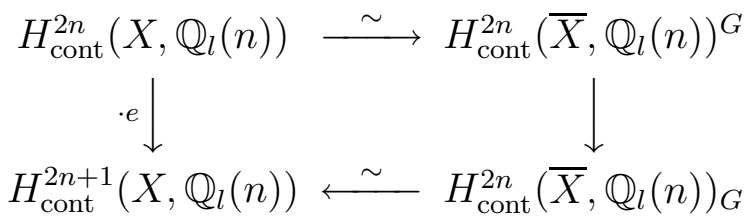

where the right vertical map is the natural one and the horizontal maps come from the (degenerating) Hochschild-Serre spectral sequence. Here, $G=\operatorname{Gal}\left(\overline{\mathbb{F}}_{p} / \mathbb{F}_{p}\right)$ and $\bar{X}=X \otimes_{\mathbb{F}_{p}} \overline{\mathbb{F}}_{p}$.

So, Conjecture 3.2 implies that the maps

$$
\begin{gathered}
C H^{n}(X) \otimes \mathbb{Q}_{l} \rightarrow H_{\text {cont }}^{2 n}\left(\bar{X}, \mathbb{Q}_{l}(n)\right)^{G} \\
H_{\text {cont }}^{2 n}\left(\bar{X}, \mathbb{Q}_{l}(n)\right)^{G} \rightarrow H_{\text {cont }}^{2 n}\left(\bar{X}, \mathbb{Q}_{l}(n)\right)_{G}
\end{gathered}
$$

are isomorphisms. The surjectivity of the first map is the cohomological Tate conjecture; its injectivity means that homological equivalence agrees with rational equivalence, and the bijectivity of the second map is condition $S^{n}(X)$ of [27].

Conversely, arguing backwards, it is easy to check that (i), (ii) and (iii) together imply that (3.1) is an isomorphism for all $q$.

Proof that Conjecture $3.1 \Longleftrightarrow$ Conjecture 3.2. By Lemma 3.8 and Proposition 3.9, it is sufficient to prove that Conjecture 3.1 is equivalent to the conditions (i)-(iii) of Proposition 3.9 for all smooth projective $X$ and all $n \geq 0$. Consider the following conditions, for all smooth, projective $X$ :

(1) The cohomological Tate conjecture;

(2) Condition $S^{n}(X)$ for all $n \geq 0$;

(3) Equality of homological and numerical equivalences.

(4) The zeta-theoretic Tate conjecture (part b) of Conjecture 3.1). 
By $[27$, th. 2.9], one has $(1)+(2) \Longleftrightarrow(1)+(3) \Longleftrightarrow(4)$. Therefore, conditions (ii) and (iii) of Proposition 3.7 are equivalent to Conjecture 3.1. On the other hand, by [8, Th. 3.3], Conjecture 3.1 implies the Beilinson-Parshin conjecture: $K_{i}(X) \otimes \mathbb{Q}=0$ for all smooth projective $X$ and all $i>0$. But this condition is equivalent to condition (i) of Proposition 3.7, cf. Theorem $1.3 \mathrm{~h}$ ). The proof is complete.

3.10. Remark. One would like to use Gabber's rigidity theorem [7] to prove Conjecture 3.3 by proving that $\mathbb{Q}_{l}(n)^{c}$ is smoothable (compare Definition 2.31 and Theorem 2.34). The obstruction is that continuous étale cohomology does not commute with direct limits.

\section{REFERENCES}

[1] S. Bloch Algebraic cycles and higher K-theory, Adv. Math. 61 (1986), 267-304.

[2] S. Bloch The moving lemma for higher Chow groups, J. Algebraic Geom. 3 (1994), 537-568.

[3] A.K Bousfield, D.M. Kahn Homotopy limits, completions and localizations, Lect. Notes in Math. 304, Springer, 1971.

[4] M. Böckstedt, A. Neeman Homotopy limits in triangulated categories, Compositio Math. 86 (1993), 209-234.

[5] J.-L. Colliot-Thélène, J.-J. Sansuc, C. Soulé Torsion dans le groupe de Chow de codimension 2, Duke Math. J. 50 (1983), 763-801.

[6] J.-L. Colliot-Thélène, R. T. Hoobler, B. Kahn The Bloch-Ogus-Gabber theorem, Fields Institute for Research in Mathematical Sciences Communications Series 16, A.M.S., 1997, 31-94.

[7] O. Gabber Affine analog of the proper base change theorem, Israel J. Math. 87 (1994), 325-335.

[8] T. Geisser Tate's conjecture, algebraic cycles and rational K-theory in characteristic $p, K$-theory 13 (1998), 109-122.

[9] T. Geisser, M. Levine, The K-theory of fields in characteristic $p$, Invent. Math. 139 (2000), 459-493.

[10] T. Geisser, M. Levine, The Bloch-Kato conjecture and a theorem of SuslinVoevodsky, J. reine angew. Math. 530 (2001), 55-103.

[11] M. Gros Classes de Chern et classes de cycles en cohomologie de Hodge-Witt logarithmique, Mém. Soc. Math. France 21 (1985).

[12] M. Gros, N. Suwa La conjecture de Gersten pour les faisceaux de Hodge-Witt logarithmiques, Duke Math. J. 57 (1988), 615-628.

[13] U. Jannsen Continuous étale cohomology, Math. Ann. 280 (1988), 207-245.

[14] P.A. de Jong Smoothness, semi-stability and alterations, Publ. Math. IHES 83 (1996), 51-93.

[15] B. Kahn A sheaf-theoretic reformulation of the Tate conjecture, preprint of the Institute of Mathematics of Jussieu no 150 (1998).

[16] B. Kahn Calculations in étale cohomology, preprint, 2001.

[17] B. Kahn Motivic cohomology, l-adic cohomology and the Tate conjecture, in preparation.

[18] K. Kato Milnor's K-theory and the Chow group of zero-cycles, Contemp. Math. 55 (I), AMS, 1986, 241-253. 
[19] K. Kato Galois cohomology of complete discrete valuation fields, Algebraic Ktheory, Part II (Oberwolfach, 1980), pp. 215-238, Lecture Notes in Math., 967, Springer, 1982.

[20] M. Levine Bloch's higher Chow groups revisited, Astérisque 226 (1994), 235320.

[21] M. Levine Mixed motives, Mathematical Surveys and Monographs 57, AMS, Providence, 1998.

[22] J. S. Milne Values of zeta functions of varieties over finite fields, Amer. J. Math. 108 (1986), 297-360.

[23] A. Nesterenko, A. Suslin Homology of the general linear group and Milnor K-theory (in Russian), Izv. Akad. Nauk SSSR 53 (1989), 121-146. English translation: Math. USSR Izv.

[24] C. Soulé Opérations en K-théorie algébrique, Can. Math. J. 37 (1985), 488550.

[25] A. Suslin Higher Chow groups and étale cohomology, in Cycles, Transfers, and Motivic Cohomology Theories, Annals of Math Studies 143, Princeton Univ. Press, Princeton, 2000.

[26] A. Suslin, V. Voevodsky Bloch-Kato conjecture and motivic cohomology with finite coefficients, The arithmetic and geometry of algebraic cycles (Banff, AB, 1998), 117-189, NATO Sci. Ser. C Math. Phys. Sci., 548, Kluwer Acad. Publ., Dordrecht, 2000.

[27] J. Tate Conjectures on algebraic cycles in l-adic cohomology, in Motives, Proc. Symposia Pure Math. 55 (1), AMS, 1994, 71-83.

[28] R.W. Thomason Le principe de scindage et l'inexistence d'une K-théorie de Milnor globale, Topology 31 (1992), 571-588.

[29] R.W. Thomason Algebraic K-theory and étale cohomology, Ann. Scient. Éc. Norm. Sup. 13 (1985), 437-552.

[SGA4] M. Artin, A. Grothendieck, J.-L. Verdier Théorie des topos et cohomologie étale des schémas (SGA4), Lect. Notes in Math. 269, 270 and 305, Springer, 1972.

[SGA41/2] P. Deligne Cohomologie étale (SGA41/2), Lect. Notes in Math. 569, Springer, 1977.

Institut de Mathématiques de Jussieu, Université Paris 7, Case 7012, 75251 Paris Cedex 05, France

E-mail address: kahn@math.jussieu.fr 